\title{
Can linear transportation infrastructure verges constitute a habitat and/or a corridor for insects in temperate landscapes? A systematic review
}

Anne Villemey ${ }^{1 *} \mathbb{D}$, Arzhvaël Jeusset ${ }^{1}$, Marianne Vargac ${ }^{1}$, Yves Bertheau $^{2,3}$, Aurélie Coulon ${ }^{2,4}$, Julien Touroult ${ }^{1}$, Sylvie Vanpeene ${ }^{5}$, Bastien Castagneyrol ${ }^{6,7}$, Hervé Jactel ${ }^{6,7}$, Isabelle Witte ${ }^{1}$, Nadine Deniaud ${ }^{8}$, Frédérique Flamerie De Lachapelle ${ }^{9}$, Emmanuel Jaslier ${ }^{9}$, Véronique Roy ${ }^{8}$, Eric Guinard ${ }^{10}$, Eric Le Mitouard $^{10}$, Vanessa Rauel ${ }^{10}$ and Romain Sordello ${ }^{1}$

\begin{abstract}
Background: The role of linear transportation infrastructures (roads, railways, oil and gas pipelines, power lines, rivers and canals) in fragmenting natural habitats is fully acknowledged. Up to now, the potential of linear transportation infrastructures verges (road and railway embankments, strips of grass under power lines or above buried pipelines, or waterway banks) as habitat or corridor for biodiversity, remains controversial. In a context of decreasing natural habitats, the opportunities of anthropogenic areas for contributing to wildlife conservation have to be considered. The present paper is the first synthesis of evidence about the potential of linear transportation infrastructure verges as corridor and/or habitat for insects in temperate landscapes.
\end{abstract}

Methods: A systematic literature survey was made using two online publication databases, a search engine and by sending a call for literature to subject experts. Identified articles were successively screened for relevance on titles, abstracts and full texts using criteria detailed in an a priori protocol. We then used six specific questions to categorize and to critically appraise the retained studies. These questions encompassed the potential of verges as habitats and corridors for insects, and the effects of management and landscape context on these potentialities. A user-friendly database was created to sort the studies with low and medium susceptibility to bias. We used these studies to synthesize results of each specific question in a narrative synthesis. Finally, studies that met the meta-analysis requirements were used for a quantitative synthesis.

Results: Our searches identified 64,206 articles. After critical appraisal, 91 articles that reported 104 studies were included in our review. Almost all of them had "control-impact" design, only two studies used "before-after-controlimpact" design, and one study used "before-after" design. In some cases, artificialization of transportation infrastructures lowered insect biodiversity while vegetation restoration had a moderate positive effect; the trend remained unclear for mowing/grazing practices. Urbanization and agriculture in the surroundings tended to lower the biodiversity hosted by verges, while natural and forested areas tended to promote it. No study dealt with the influence of management or surrounding landscape on insect dispersal along the verge. The small number of studies that compared the dispersal along verges and in habitats away from transportation infrastructures, together with the

\footnotetext{
*Correspondence: anne.villemey@gmail.com

1 UMS 2006 PatriNat, Centre National de la Recherche Scientifique (CNRS),

Muséum national d'Histoire naturelle (MNHN), Agence Française pour la

Biodiversité (AFB), 75005 Paris, France

Full list of author information is available at the end of the article
} 
inconsistencies of their results, prevented us from drawing conclusions. Meta-analyses were performed on 709 cases from 34 primary studies that compared biodiversity in verges vs. other habitats. Overall insect species richness did not differ between LTI verges and compared habitats. Globally, insect abundance seemed higher on LTI verges than in compared habitats, a result driven by the higher abundance of pollinators and primary consumers on non-highway road verges than in habitats away from roads.

Conclusions: A major knowledge gap regarding the potential of linear transportation infrastructure verges as corridors for insects has been identified. Thus, we encourage more research on this topic. Infrastructure practitioners could benefit from our results about linear transportation infrastructure verges as habitat for certain taxa and about the impact of their management practices on insect abundance and species richness.

Keywords: Coleoptera, Diversity, Green infrastructure, Hymenoptera, Intervention, Invertebrates, Lepidoptera, Movement, Right of way, Roadside

\section{Background}

For the last decades, human activities have resulted in a worldwide erosion of biodiversity [1]. The loss of natural habitats associated with landscape urbanization and fragmentation stands among the reasons for these extinctions [2]. Landscape fragmentation includes the loss and the split of natural habitats into multiple isolated patches of reduced size $[3,4]$. The theory of island biogeography predicts that the species richness of a habitat patch decreases with its reduction in size and its increasing isolation [5]. The breaking apart of habitats per se has immediate and time-delayed effects on biodiversity [6], independently of habitat losses [7]. In the short term, fragmentation has negative consequences for habitat selection, abundance and species diversity [8-10]. In the long term, fragmentation limits or disrupts migration and dispersal of juveniles and adults, which can substantially impair meta-population dynamics [11, 12]. Individual species are then exposed to various stochastic threats, leading in some cases to extinction cascades [13] and extinction debts [14]. Thus habitat fragmentation ultimately leads to ecosystem decay [15].

Both urbanization and the development of linear transportation infrastructures (LTIs) are causes of landscape fragmentation $[16,17]$. LTIs lead to a net disruption of the natural habitats that they cross, splitting them into several distinct patches [18]. LTIs induce an indirect decrease of abundance and species diversity in the surrounding landscape $[19,20]$. They also cause direct animal mortality due to vehicle, fence or catenary line collisions, electrocutions and drownings of individuals attempting to cross the infrastructures [21-23]. Over several generations, LTIs have also been shown to lead to a genetic isolation of populations [24-26].

To overcome fragmentation issues, corridors have received an increasing interest in conservation biology [27]. However, well-designed studies to evaluate corridor effectiveness through demography, genetics or dispersal investigations are not so common [28]. In the last decades, scientists and policymakers have paid attention to the potential of blue-green infrastructures, i.e. networks of ecological land and aquatic continuities, aiming to decrease habitat fragmentation in the short term [29]. Moreover, in the long term, maintaining a network of ecological corridors could mitigate the effects of climate change through enhanced dispersal of species to newly suitable areas [30]. In the context of such biodiversity losses, the potential of anthropogenic areas for preserving nature deserves to be considered [31].

Up to now, studies about habitat fragmentation have considered LTIs transversally, i.e. they have focused on biodiversity dispersal flows perpendicular to LTIs. Yet, the potential of LTIs verges considered longitudinally, i.e. focusing on biodiversity dispersal flows parallel to LTIs, remains unclear. LTIs are generally made of a transportation lane (road, railway, pipeline, powerline, river or canal) and of verges (road and railway embankments, strips of grass under power lines or above buried pipelines, or waterway banks, etc.). A verge is a strip along, between, above or below the carriageway(s), inside the LTI boundaries, not directly used for transportation and managed by the LTI owner. In most cases, verges are covered by vegetation and may potentially constitute seminatural habitats. This vegetation can either be "natural", dominated by exotic species or mono-specific. It is thus of interest to assess whether, despite their fragmenting effect, LTI verges could contribute longitudinally to a network of blue-green infrastructures and thus to biodiversity conservation.

At first sight, the studies that have considered LTI verges longitudinally seem to have provided contrasted results. For instance, according to Bolger et al. [32], revegetated highway rights-of-way could serve as ecological corridors for Californian native rodents and fragmentation-tolerant bird species. On the contrary, Benítez-López et al. [33] showed that population 
densities of mammals and birds, but not raptors, declined with their proximity to infrastructures. In addition, a verge management practice can be beneficial for some species and detrimental for other ones [34, 35]. Finally, some management practices that would be positive for biodiversity may be impossible to perform for safety reasons [36].

As far as we know, there is thus no consensus in the scientific community regarding the topic. Therefore, LTI managers and the French ministry in charge of the Environment requested a systematic review, taking into account all accessible studies and synthesizing their results. Such a review might be of interest to LTI managers and policy makers by identifying the conditions under which LTI verges could play a role of habitat and/or corridor for biodiversity. Previous reviews on the influence of one specific type of LTI on biodiversity have already been published $[37,38]$ but they do not fulfill the standard of systematic review [39]. To date, no systematic review addressed the potential role LTIs may play on biodiversity as corridors or habitats.

In France, the concept of green infrastructures led to the development of a public policy project named "Trame Verte et Bleue" in 2007. This project was developed by the "Ministère de l'Environnement de l'Énergie et de la Mer" (MEEM, Ministry in charge of the environment). Accordingly, French administrative regions have identified ecological networks and are currently developing action plans for preserving and restoring these continuities. This issue has also to be considered in local urban planning. Through different spatial scales, various stakeholders work on the issue of habitat fragmentation.

As the LTI network is very dense in France, LTI managers might substantially contribute to ecological networks. For instance, the French road network, with over a million kilometer long, is the longest $(1 / 4$ of the European network) and one of the densest $\left(1.77 \mathrm{~km} / \mathrm{km}^{2}\right)$ of the European Union. As a comparison, Spain, which has an area close to the one of France, has a road density six times lower $\left(0.32 \mathrm{~km} / \mathrm{km}^{2}\right)$. SNCF Réseau manages more than $30,000 \mathrm{~km}$ of railway lines in use (out of 50,000 km), which constitutes the longest railway network of Europe. Meanwhile, such a dense LTI network means a considerable inherent surface of verges. The total area of French road verges is indeed estimated to $4500 \mathrm{~km}^{2}$, thus superior to the total area of $3450 \mathrm{~km}^{2}$ of the seven terrestrial national parks [40]. Accordingly, LTI verges could substantially contribute to green infrastructures. Aware of these issues, several French LTI managing companies have gathered in an informal group, named "Club des Infrastructures Linéaires \& Biodiversité" (CILB), aiming at acting for biodiversity conservation. Among the members of the CILB, motorway, railway, power line, pipeline and waterway French stakeholder companies ${ }^{1}$ decided to evaluate whether their LTI verges could contribute to blue-green infrastructures for improving the management of these verges for that purpose.

The systematic review was assumed to be a relevant scientific method for providing a sound answer to this practical questioning from LTI managers. The French ministry in charge of the environment undertook a call for tender for a systematic review. This call was developed through its research incentive program relative to transportation ecology, named "Infrastructures de Transport Terrestre, Écosystèmes et Paysage" (ITTECOP), with the help of the CILB and the "Fondation pour la Recherche sur la Biodiversité" (FRB), a French foundation supporting research in biodiversity.

The "Muséum national d'Histoire naturelle" (MNHN) was then chosen for conducting the project. The review team also gathered teams of the "Institut national de recherche en sciences et technologies pour l'environnement et l'agriculture" (Irstea), the University of Pierre and Marie Curie (UPMC), the "Centre d'études et d'expertise sur les risques, l'environnement, la mobilité et l'aménagement" (Cerema) and of the "Institut national de la recherche agronomique" (Inra).

The protocol of the systematic review which covers all the kinds of biodiversity has previously been published [41]. However, due to the very large number of articles collected, we decided to split the review process by taxa. Therefore, this first systematic review focuses on insects, as they constitute an essential functional group which is often overlooked in reviews about LTIs [33, 38, 42].

\section{Objective of the review}

The objective of the review is to assess if LTI verges can provide habitats for insects and if they can be used as corridors by insects. The review also aims at assessing the effect of management practices (mowing, grazing, etc.), and of surrounding landscape on the potential of LTI verges for insect biodiversity. The review first lists the relevant studies in a database. We then used narrative syntheses to summarize the evidence about the potential of LTI verges as corridors or habitats for insects and the influence of verge management and surrounding landscape. Finally, whenever the nature of primary studies allowed this quantitative approach, we used meta-analyses to quantify the effect of LTIs on insect biodiversity and test whether variability in insect response differed with species characteristics and could be explained by key moderators such as LTI type.

\footnotetext{
${ }^{1}$ Réseau Ferré de France, Voies Navigables de France, Réseau de Transport d'Électricité, GRT Gaz, Transport et Infrastructures Gaz France and Électricité Réseau Distribution France.
} 


\section{Broad review question}

The broad review question is: can linear transportation infrastructure verges constitute habitats and/or corridors for insects in temperate landscapes?

\section{Specific synthesis questions}

We split the above broad review question into six more specific synthesis questions detailed in Table 1 . This split was used during the critical appraisal of the studies and the synthesis of the evidence (see below). Questions 2, 4, 5 and 6 are Population, Exposure, Comparator, Outcome (PECO) questions, where Exposure is the LTI verge in questions 2 and 4 and the type of surrounding landscape in questions 5 and 6. Questions 1 and 3 are Population, Intervention, Comparator, Outcome (PICO) questions, where Intervention is the management practices of LTI verge.

\section{Components of the broad review question}

The present study does not consider the transversal effects of LTIs on biodiversity, such as barrier effect, which have already been documented [19, 43, 44]. It focuses exclusively on the longitudinal effects of LTI verges on insect diversity and dispersal. Potential positive (role of corridor/habitat, etc.), neutral, as well as negative (dispersal of invasive species, sink habitat effect, resistance to insect dispersal, etc.) longitudinal effects of LTI verges on insects are considered.

Table 1 Details of the six specific synthesis questions of the review

\begin{tabular}{|c|c|c|}
\hline Number & Details & Short question \\
\hline Question 1 & $\begin{array}{l}\text { Which LTI verge management } \\
\text { practices increase, decrease } \\
\text { or have no effect on insect } \\
\text { biodiversity? }\end{array}$ & $\begin{array}{l}\text { Habitat/management } \\
\text { practices }\end{array}$ \\
\hline Question 2 & $\begin{array}{l}\text { Is the insect biodiversity of LTI } \\
\text { verges equal to, higher, or } \\
\text { lower than the biodiversity of } \\
\text { habitats away from the LTIs? }\end{array}$ & $\begin{array}{l}\text { Habitat in LTI verges } \\
\text { vs. at proximity }\end{array}$ \\
\hline Question 3 & $\begin{array}{l}\text { Which LTI verge management } \\
\text { practices increase, decrease } \\
\text { or have no effect on insect } \\
\text { dispersal? }\end{array}$ & $\begin{array}{l}\text { Dispersal/manage- } \\
\text { ment practices }\end{array}$ \\
\hline Question 4 & $\begin{array}{l}\text { Is insect dispersal on LTI verges } \\
\text { equal to, higher, or lower } \\
\text { than their dispersal in habi- } \\
\text { tats away from the LTI? }\end{array}$ & $\begin{array}{l}\text { Dispersal in LTI verges } \\
\text { Vs. at proximity }\end{array}$ \\
\hline Question 5 & $\begin{array}{l}\text { Is the insect biodiversity of LTI } \\
\text { verges dependent on the } \\
\text { surrounding landscape? }\end{array}$ & $\begin{array}{l}\text { Habitat/surrounding } \\
\text { landscape }\end{array}$ \\
\hline Question 6 & $\begin{array}{l}\text { Is insect dispersal on LTI verges } \\
\text { dependent on the surround- } \\
\text { ing landscape? }\end{array}$ & $\begin{array}{l}\text { Dispersal/surrounding } \\
\text { landscape }\end{array}$ \\
\hline
\end{tabular}

a The spatial scale of the surrounding landscape can range from the land use directly adjacent to the LTI verge, to radii of hundreds of meters
Table 2 Description of the PECO/PICO items of the primary question

\begin{tabular}{|c|c|}
\hline Population & $\begin{array}{l}\text { All insect species and communities of the temperate } \\
\text { climatic zone }\end{array}$ \\
\hline Exposure & $\begin{array}{l}\text { LTI verges (road, railway, power line and pipeline verges } \\
\text { and waterway banks) }\end{array}$ \\
\hline Intervention & $\begin{array}{l}\text { Management practices or human-induced disturbances } \\
\text { of LTI verges }\end{array}$ \\
\hline Comparator & $\begin{array}{l}\text { Both temporal and spatial comparators, including but not } \\
\text { restricted to: } \\
\text { Temporal comparators } \\
\text { Ecosystem present before infrastructure construction } \\
\text { Verge before management intervention } \\
\text { Spatial comparators } \\
\text { Nearby habitats away from LTIs } \\
\text { Unmanaged verges or verges managed with a differ- } \\
\text { ent practice }\end{array}$ \\
\hline Outcome & $\begin{array}{l}\text { All outcomes relating to corridor or habitat assessment, } \\
\text { including but not restricted to, species dispersal, species } \\
\text { richness, abundance and community composition }\end{array}$ \\
\hline
\end{tabular}

a The Köppen-Geiger Climate Classification was used to identify articles with a study zone in the temperate climate. As the funders of the study are interested in western Europe, the $\mathrm{Cfa}, \mathrm{Cfb}, \mathrm{Cfc}, \mathrm{Csa}, \mathrm{Csb}$ and $\mathrm{Csc}$ temperate zones were included in the scope of our study. See http://people.eng.unimelb.edu.au/ mpeel/koppen.html (accessed 17 November 2015) for the GoogleEarth layers of the Köppen-Geiger Climate Classification

Table 2 displays the components of the broad review question $^{2}$ : population, exposure/intervention, comparator, outcome (PECO/PICO).

Our systematic review focused on linear transportation infrastructures, thus, we only considered verges of navigable waterways (navigable rivers and canals) as relevant exposures.

Regarding "nearby habitats away from LTIs" (comparator in Table 2), we included the comparisons of verges with both similar and dissimilar ecosystems because these comparisons support two meaningful research questions. Indeed, studies that compare LTI verges with nearby similar semi-natural habitats evaluate if verges can constitute optimal or sub-optimal habitats for species. Studies comparing LTI verges with nearby dissimilar habitats evaluate the influence of LTI construction on biodiversity at the construction site, nearby habitats being considered as proxies of the initial habitat before LTI construction.

\section{Methods}

\section{Search strategy}

We searched for literature using online publication databases and a search engine. In addition, we sent a call for literature to subject experts to collect grey literature.

\footnotetext{
${ }^{2}$ LTI managers funding the study were met at the beginning of the project to list the types of verges that they own and the management practices that they apply on those.
} 
Searches in the publication databases and in the search engine were undertaken using English terms only, but articles written in either English or French languages and found with the English search strings were included. At this stage, no date nor type of publication restrictions were applied.

\section{Online publication databases}

We first listed the databases to which the members of our review team had access.

The database selection was then based on three criteria [41]:

- topic: database(s) had to cover ecology,

- accessibility/reproducibility/sustainability: database(s) had to be accessible by the whole review team, and by researchers all over the world (as a guarantee of reproducibility and further reviewing),

- comprehensiveness: number of articles indexed in the database(s) among the 102 articles of the test list, ${ }^{3,4}$ (see Additional file 2).

These criteria led us to select two databases: Web of Science Core Collection (WOS CC, 84 articles indexed out of the 102 articles of the test list) and Zoological Records (ZR, 51 articles out of the 102 articles).

\section{Search strings}

The search terms identified by the review team are displayed in Additional file $1 .^{5}$

\footnotetext{
${ }^{3}$ We first requested experts to send us the five scientific articles that they considered the most relevant regarding our research topic. Following the e-mail request, 77 scientific articles were sent to us by 21 experts. Out of these articles, 50 were collectively assessed, by the research team, as relevant considering our scientific question. Studies that were excluded of the list mostly dealt with road-kills, habitat fragmentation, wildlife overpasses, green infrastructures in general, environmental impact assessment of LTI construction, naturalist inventories out of the temperate climatic zone and pedology of LTI verges. Those subjects were considered by the research team as subsidiary to the scientific question. On top of these 50 articles provided by experts, 23 scientific articles, known by the members of the research team to address our scientific question, were added to the list. Nevertheless, at that stage, few articles of the list were related to pipelines, waterways and railways. Thus, 29 relevant scientific articles were searched on Google Scholar in order to balance the proportion of articles of the list related to each LTI. The final list of 102 approved key articles is detailed in Additional file 2.

${ }^{4}$ E-mail addresses of experts were obtained through mailing lists in ecology (Ecodiff, Transenviro, Wftlistserv and IENE, detailed in the following endnotes) and directories owned by members of our research team. The directories contain the contact details of 1902 persons working on green infrastructures, including scientists, LTI managers and government officers. Most of these persons work in France but the directories also include 21 international contacts, coming from 14 countries.

${ }^{5}$ For all keywords listed, wildcards may be used, to allow the use of derivations of the word's root and to account for the possibility of finding a word in various spellings (English from Great Britain or from the United States) and with various endings (singular or plural).
}

We tested a first search string combining some of the search terms with Boolean operators of Web of Science Core Collection. To assess the relevance of the search string, we compared the search hits to the articles of the test list (see footnotes 3, 4, Additional file 2) identified by 25 subject experts indexed in the database. Then, we modified the search string by removing some of the search terms and including new ones, to increase the number of articles of the test list recovered [41].

A first scoping of search hits revealed that a global search string including all LTIs brought many irrelevant results linked to waterways. Consequently, we split the search into a first string concerning all LTIs except waterways and a second string specific to waterways, which reduced the total number of search hits without decreasing comprehensiveness.

For each of the non-waterway and waterway searches, we developed two different search strings in parallel (strategies 1 and 2, Table 3), reaching similar high levels of comprehensiveness. As no argument justified choosing one rather than the other, we retained both strings; we merged results and discarded duplicates. Both search strings included LTI synonyms, verge synonyms and outcomes categories, and the terms within each category were combined using the Boolean operator "OR" (Table 3). However, while strategy 2 combined each of the three categories with the Boolean operator "AND", strategy 1 combined the categories of verge synonyms and outcomes with the Boolean operator "OR" and the category of LTI synonyms to the two other categories with the Boolean operator "AND". Strategy 1 considered that neither the list of verge synonyms nor the list of ecological outcomes were exhaustive. Thus, it combined both of them with the Boolean operator "OR" hoping that articles about unlisted outcomes were found by the use of a verge synonym and conversely. Strategy 2 separated verges' and outcomes' synonyms in different strings but allowed synonyms with a broader meaning to obtain a high comprehensiveness anyway.

We applied the four search strings specified in Table 3 to the searches in the online databases (WOS CC and ZR).

\section{Search engine}

We performed internet searches using Google Scholar (https://scholar.google.fr/). Search strings had to be simplified as Google Scholar could only handle a very limited number of search terms and did not allow the use of all "wildcards". Thus, we developed a search string for each of the five LTIs (Additional file 3). Results were sorted by relevance, with the boxes "include patents" and "include citations" unchecked. For each of the five search strings, we retrieved the first 20 hits. 
Table 3 Search strings ${ }^{a, b, c}$ selected and used in wOS CC and ZR

\begin{tabular}{|c|c|c|}
\hline LTI & Strategy & Search string \\
\hline \multirow[t]{2}{*}{$\begin{array}{l}\text { Roads, railways, } \\
\text { pipelines and } \\
\text { power lines }\end{array}$} & 1 & $\begin{array}{l}\text { LTIs: ("transport* infrastructure*" OR } \\
\text { road* OR highway\$ OR motorway\$ } \\
\text { OR freeway } \$ \text { OR rail* OR pipeline\$ } \\
\text { OR powerline\$ OR"power line" OR } \\
\text { "power lines" OR "transmission line*" } \\
\text { OR "electric }{ }^{*} \text { line" OR "electric* lines" } \\
\text { OR "electric* pylon*") } \\
\text { AND } \\
\text { Verges/outcomes: (corridor\$ OR dis- } \\
\text { persal\$ OR habitat\$ OR refuge\$ OR } \\
\text { "right* of way*" OR verge\$ OR abun- } \\
\text { dance OR richness OR composition\$ } \\
\text { OR *diversity OR communit*) }\end{array}$ \\
\hline & 2 & $\begin{array}{l}\text { LTIs: (road* OR highway* OR motorway* } \\
\text { OR rail* OR "transmission line* cor- } \\
\text { ridor*" OR powerline* OR pipeline* } \\
\text { OR "electric* pylon*") } \\
\text { AND } \\
\text { Verges: (corridor* OR habitat* OR verge* } \\
\text { OR right\$-of-way* OR proximity OR } \\
\text { contiguous OR line\$) } \\
\text { AND } \\
\text { Outcomes: (dispers* OR population* OR } \\
\text { communit* OR abundan* OR distri- } \\
\text { bution\$ OR "species composition*" } \\
\text { OR attendance) }\end{array}$ \\
\hline \multirow[t]{2}{*}{ Waterways } & 1 & $\begin{array}{l}\text { LTIS/verges: (riparian OR riverside\$ OR } \\
\text { riverbank\$ OR “river* *bank*" OR } \\
\text { ((waterway\$ OR canal\$ OR channel\$) } \\
\text { AND *bank*)) } \\
\text { AND } \\
\text { Outcomes: (corridor\$ OR dispersal\$ OR } \\
\text { habitat\$ OR refuge\$ OR abundance } \\
\text { OR richness OR *diversity OR compo- } \\
\text { sition\$ OR communit*) }\end{array}$ \\
\hline & 2 & $\begin{array}{l}\text { LTIs: (river* OR channel\$ OR stream\$) } \\
\text { AND } \\
\text { Verges: (riparian\$ OR *bank* OR proxim- } \\
\text { ity OR bridge\$) } \\
\text { AND } \\
\text { Outcomes: (dispers* OR communit* OR } \\
\text { richness OR diversity OR drowning } \\
\text { OR roosting OR "alien plant*") }\end{array}$ \\
\hline
\end{tabular}

a The asterisk $\left(^{*}\right)$ represents any group of characters, including no character

b The dollar sign (\$) represents zero or one character

c The quotation marks (" ") allow to look for an exact phrase

\section{Call for literature}

To retrieve grey literature, we contacted by e-mail national and international experts of transportation ecology, through the Ecodiff, ${ }^{6}$ Transenviro, Wftlistserv and
$\mathrm{IENE}^{7}$ mailing lists and by posting a call on social media (https://fr.linkedin.com/). We contacted nearly two thousand people $(\mathrm{N}=1902)$ by individual email. Organizations funding the systematic review also provided us with their unpublished reports.

\section{Specialist websites}

Due to the large number of documents received in response to the call for grey literature $(\mathrm{N}=495)$, we considered searches on specialist websites as redundant. Actually, most of the contacted experts are part of the organizations whose specialist websites had been identified in the protocol of the present review [41]. We are thus confident that most of the grey literature from the specialist websites identified in the protocol was sent to us by e-mail.

\section{Screening and inclusion criteria Scientific articles}

We assessed scientific articles collected in online publication databases for inclusion at three successive levels: first on titles, second on abstracts and third on full-texts. At each stage, in case of uncertainty, we retained articles for assessment at the following stage. Articles about non insects species were put aside during the workflow of title, abstract and full-text screenings.

At each stage, article eligibility was based on a list of selection criteria. At the title screening stage, these criteria encompassed both the subject (ecology and related disciplines) and the population of the article (Table 4). The same criteria were used at the abstract screening stage, to which we added criteria regarding the exposure/ intervention, the comparator, the outcomes or the study type (Table 5). Articles without abstract were excluded. Finally, the same criteria as for the abstract stage were used for the full-text screening stage, to which we added inclusion/exclusion criteria specific to the full-text stage regarding the language, the climate, the type of publication or the specific questions covered (Table 6).

\section{Grey literature}

Grey literature, which does not comply with scientific publishing standards, was assessed on title and full-text (with the same criteria as those listed above for published literature). Ph.D. theses were first screened to determine their eligibility. Each potentially relevant chapter/article was then treated separately as a unique full-text.

\footnotetext{
${ }^{6}$ Ecodiff is a French mailing list about ecology and evolution, which counts around 7000 subscribers. http://www.sfecologie.org/ecodiff/, Accessed 22 September 2015.
} 
Table 4 List of exclusion/inclusion criteria at the stage of title screening

\begin{tabular}{|c|c|}
\hline Include & Exclude \\
\hline \multicolumn{2}{|l|}{ For all LT/s } \\
\hline $\begin{array}{l}\text { Articles dealing only partially with the role of habitat or corridor of the } \\
\text { verges }\end{array}$ & $\begin{array}{l}\text { Studies regarding green infrastructures in general without considering the } \\
\text { specific case of LTls }\end{array}$ \\
\hline $\begin{array}{l}\text { Articles regarding invasive species if the role of corridor or habitat of } \\
\text { verges is mentioned }\end{array}$ & $\begin{array}{l}\text { Studies regarding overpasses/underpasses or fragmentation due to LTIs } \\
\text { considered transversally, without considering the roles of habitat and }\end{array}$ \\
\hline Articles regarding soil biodiversity & corridor of verges \\
\hline $\begin{array}{l}\text { Articles dealing with the effects of chemical, noise or light pollution on } \\
\text { verge biodiversity (even if the pollution comes from the infrastructure }\end{array}$ & $\begin{array}{l}\text { Studies regarding paleontology, phylogenetics, phylogeography and tax- } \\
\text { onomy (including studies describing newly discovered species) }\end{array}$ \\
\hline itself) & Genetic studies without any relation to a natural habitat (in particular biodi- \\
\hline Articles out of the temperate climatic zone (this criteria is assessed at the & versity meta-genomics studies) \\
\hline full-text reading stage) & Pedological studies without any relation to biodiversity \\
\hline $\begin{array}{l}\text { Articles regarding wildfires (they are assessed at the full-text reading } \\
\text { stage) }\end{array}$ & $\begin{array}{l}\text { Studies regarding medicine, toxicology or chemical, noise or light pollution } \\
\text { without any relation to biodiversity }\end{array}$ \\
\hline \multicolumn{2}{|l|}{ Specifically for fluvial LTIs (waterways) } \\
\hline $\begin{array}{l}\text { Articles whose title mentions the words floodplain, riparian, wetland, sea- } \\
\text { sonal pond, intermittent stream or spawning (in which case the article is } \\
\text { considered to deal with the semi-aquatic part of the river, that is to say } \\
\text { the banks, emerged during the dry season and immersed during the } \\
\text { wet season, which is part of the scope of the review) }\end{array}$ & $\begin{array}{l}\text { Articles regarding exclusively aquatic species, except if the title mentions } \\
\text { the words floodplain, riparian, wetland, seasonal pond, intermittent } \\
\text { stream or spawning (in which case the article is considered to deal with } \\
\text { the lateral part of the river, that is to say the banks, sometimes immersed } \\
\text { other times emerged, which is part of the scope of the review) }\end{array}$ \\
\hline Articles regarding amphibious species & Articles regarding lakes and islands or sand banks in the middle of rivers \\
\hline Articles regarding seed dispersal through waterway flow (hydrochory) & Articles regarding river debris (organic matter, tree trunks, underwater \\
\hline Articles regarding the role of waterway banks in animal drownings & leaves decomposition, except if the article deals with the submerged part \\
\hline $\begin{array}{l}\text { Articles recommending management actions to perform under bridges } \\
\text { (hanging bat roosting boxes for instance) }\end{array}$ & $\begin{array}{l}\text { of the bank, etc.) } \\
\text { Articles regarding drownings without any relation to the role of habitat of }\end{array}$ \\
\hline Articles regarding streams (they are assessed at the full-text reading stage) & the banks \\
\hline \multicolumn{2}{|c|}{ Specifically for non-fluvial LTIs (roads, railways, power lines, pipelines) } \\
\hline $\begin{array}{l}\text { Articles regarding the role of verges in animal collisions } \\
\text { Articles recommending verge management actions to perform (including } \\
\text { fencing to avoid collisions) }\end{array}$ & $\begin{array}{l}\text { Articles regarding animal collisions without any relation with the role of } \\
\text { habitat or corridor of the verges }\end{array}$ \\
\hline
\end{tabular}

Table 5 List of inclusion criteria at the stage of abstract screening

\begin{tabular}{|c|c|}
\hline Type of criteria & Description \\
\hline Relevant population(s) & $\begin{array}{l}\text { All insect biodiversity (at the species, community and ecosystem level) of the temperate climatic zone }{ }^{\mathrm{a}} \text {, including } \\
\text { exotic invasive species }\end{array}$ \\
\hline Types of exposure/intervention & $\begin{array}{l}\text { Any article exposing biodiversity to a LTI verge (road, railway, power line or pipeline verges or waterway banks), to } \\
\text { a LTI verge management (mowing, pesticide spreading, pruning, planting, fence laying, beehive setting up, bird } \\
\text { nesting box and bat roosting box hanging, reptile artificial refuge setting up, etc.) or to a LTI verge disturbance } \\
\text { (chemical, air, noise and light pollution and wildfires) }\end{array}$ \\
\hline Types of comparator & Unexposed/intervention-free control site or before-exposure/before-intervention control site \\
\hline Types of outcome & $\begin{array}{l}\text { All outcomes relating to corridor and habitat assessment or effects of verge management, such as dispersal (includ- } \\
\text { ing species invasions, hydrochory and seed dispersal by vehicles), species richness, Shannon index, Simpson } \\
\text { index, beta diversity, community composition and abundance of different taxonomic or functional groups of } \\
\text { organisms }\end{array}$ \\
\hline Types of study & $\begin{array}{l}\text { All type of studies should be included apart from modelling (theoretical) articles, articles making recommendations } \\
\text { without making experimentation and articles making experimentations in laboratory conditions }\end{array}$ \\
\hline
\end{tabular}

a The Köppen-Geiger Climate Classification used to identify articles with a study zone in the temperate climate. As the funders of the study are interested in western Europe, the $\mathrm{Cfa}, \mathrm{Cfb}, \mathrm{Cfc}, \mathrm{Csa}, \mathrm{Csb}$ and Csc temperate zones were included in the scope of our study. See http://people.eng.unimelb.edu.au/mpeel/koppen.html (accessed 17 November 2015) for the GoogleEarth layers of the Köppen-Geiger Climate Classification

\section{Test of consistency of reviewers' inclusion/exclusion decisions}

Before the onset of screening, the reviewers taking part in the assessment process tested the consistency of their inclusion/exclusion decisions. For each of the sets of articles about waterways and other LTIs and for each of the title and abstract screening stages, we randomly selected a sample of articles and each of the reviewers screened them independently of each other. As more than two reviewers took part in each article inclusion assessment, we computed Randolph's Kappa coefficients in $R$ 3.3.2 [45]. A coefficient of 0.6 was set as the minimal 
Table 6 List of inclusion criteria at the full-text stage

\begin{tabular}{|c|c|}
\hline Type of criteria & Description \\
\hline Language & Full text written in English or French \\
\hline Climate & Articles with study zone(s) of the temperate climate ${ }^{a}$ \\
\hline Type of publication & Articles different from editorial material, meeting abstracts, news items and review \\
\hline Comparator & Articles with control/compared site (not observational studies) \\
\hline Road type & Articles with paved road (not unpaved road, path, gravel road, forest road) \\
\hline Waterway type & Articles with stream order above three, canals or rivers ${ }^{b}$ \\
\hline Specific questions & Articles that give relevant results for the six specific synthesis questions detailed in Table 1 \\
\hline \multicolumn{2}{|c|}{$\begin{array}{l}\text { The Köppen-Geiger Climate Classification used to identify articles with a study zone in the temperate climate. As the funders of the study are interested in western } \\
\text { Europe, the Cfa, Cfb, Cfc, Csa, Csb and Csc temperate zones were included in the scope of our study. See http://people.eng.unimelb.edu.au/mpeel/koppen.html } \\
\text { (accessed } 17 \text { November 2015) for the GoogleEarth layers of the Köppen-Geiger Climate Classification } \\
\text { b Only navigable rivers and canals are transportation infrastructures, unfortunately, this information is rarely provided. Thus, we included all articles with stream order } \\
\text { above three, canals and rivers; we excluded all articles with stream order equal or below three and articles with no information }\end{array}$} \\
\hline
\end{tabular}

acceptable level of estimated agreement between reviewers. When the coefficient was lower than 0.6, reviewers in disagreement discussed until choosing common selection criteria. Then, they re-tested the consistency of their inclusion/exclusion decisions on a new set of articles and computed Randolph's Kappa coefficients. This operation was repeated until reaching a coefficient superior to 0.6. The final scores ranged from 0.667 to 0.780 $($ mean $=0.740)$. We performed this test of consistency on the articles obtained from Web of Science Core Collection publication database, which was the first database processed. We did not repeat this process for the Zoological Records publication database, but since the same reviewers performed both database screenings, we are confident on the consistency of their inclusion/exclusion decisions.

Although it is good practice to perform a test of consistency of reviewers' inclusion/exclusion decisions at the full text stage we were unable to do this due to logistical constraints and time limitation.

We took care that reviewers never had to screen or critically appraise articles they authored by themselves.

\section{Moderators potentially accounting for heterogeneity in the insect responses to LTI verges}

We recorded the following potential effect modifiers as stated in the protocol of the present review [41]:

- geographic location,

- biological group of insects studied,

- site characteristics: type of LTI, type of habitat of the verge and type of habitat of the compared site,

- verge management practices (mowing, grazing, vegetation burning, pesticide use, etc.),

- comparator type (spatial/temporal, etc.),

- selection of sample location (randomization, fixed distances or grids versus opportunistic sampling).
Although identified as a potential reason for heterogeneity in the protocol of the present review [41], we eventually considered the absence of replicates as a important source of bias. Accordingly, those articles without replicates were discarded during critical appraisal.

\section{Study validity assessment}

In order to develop the critical appraisal of the studies included following full-text screening, eight external experts in landscape connectivity and transportation ecology and seven scientists of the review team were gathered and consulted during a 1-day workshop [41]. During the workshop, we discussed the gold standard protocol of an ideal study answering our primary question with unlimited resources. The criteria used to assess the relevance and the susceptibility to bias were based on these discussions.

We considered that a study was not relevant to the purpose of the review, and thus discarded it, if:

- the comparator was inappropriate (comparison between different seasons, comparator difficult to interpret for the purposes of this review, etc.),

- the results included biological groups and/or exposures that were not under the scope of the review, with no possibility to extract results scoping the review (e.g. results combining insects and noninsects: macro-invertebrates, results combining paths and paved roads).

Since we checked data redundancy (data already published in another article included in the review) during critical appraisal, we added this factor as an exclusion cause, although it is not strictly a quality criterion.

We considered that a study was unreliable because of a high susceptibility to bias, and therefore excluded it from the review, if there was: 
- a total absence of replications,

- an inadequate methodology (for example for question \#4: statistical analysis of movement data that did not allow to distinguish LTI verges from other habitats),

- a method description strongly insufficient (i.e. when it is not possible to know where the sampling was done: within or without LTI verges),

- major confounding factors.

We considered the studies that had the characteristics detailed below as having medium susceptibility to bias:

- absence of transparent and systematic procedure for the selection of sample plot location (i.e. randomization, fixed distances, grids),

- comparator-intervention and before-after-intervention study designs (as opposed to before-after-comparator-intervention study designs) for the specific questions involving verge management (questions 1 and 3),

- absence of true spatial replication of the study (for example study with repetition of measures on a unique site),

- attrition bias (difference in the loss of samples between control and treatment),

- method description slightly insufficient (some minor details were missing but did not challenge our understanding of the methods).

Studies that were not considered to have high and medium susceptibility to bias were considered to have low susceptibility to bias.

For articles dealing with more than one specific question (Table 1), we performed critical appraisal for each question separately, that we considered being different studies.

The critical appraisal was performed as follows: first, each study was critically appraised by one reviewer. Then, a second reviewer critically appraised again the uncertain cases. We compared conclusions of the two reviewers, and when they differed, they discussed disagreements until reaching a consensus and asked for a third reviewer if necessary.

Studies that did not display any statistical comparison between treatments and controls were included in the review. These studies can provide data for meta-analyses, but they were not included in the narrative syntheses.

\section{Study coding and data extraction}

We produced a database of the articles included after critical appraisal (low and medium susceptibility to bias). We used the coding tool displayed in Table 7. If an article dealt with more than one of our specific questions, we
Table 7 Coding tool for the included studies database

\begin{tabular}{|c|c|}
\hline Coding variable & Details/examples \\
\hline Reference & Authors, year, title, publisher \\
\hline Publication type & $\begin{array}{l}\text { Book chapter, journal paper, thesis, report, } \\
\text { etc. }\end{array}$ \\
\hline Article language & English/French \\
\hline Specific question & Question \#1, 2, 3, 4, 5 or 6 \\
\hline \multicolumn{2}{|l|}{ Study country } \\
\hline \multicolumn{2}{|l|}{ Study region(s) } \\
\hline \multicolumn{2}{|l|}{ GPS coordinates } \\
\hline Biological group(s) & Carabid, wild bees, etc. \\
\hline $\operatorname{LTI}(s)$ & $\begin{array}{l}\text { Roads/railways/power lines/pipelines/ } \\
\text { waterways }\end{array}$ \\
\hline Type of habitat of the verge & Grassland, shrubland, hedge, forest, etc. \\
\hline Comparison & $\begin{array}{l}\text { Questions \#2 and 4: type of habitat of the } \\
\text { control site } \\
\text { Questions \#1 and 3: management } \\
\text { practices (mowing, pesticide spreading, } \\
\text { pruning, planting, fence laying, beehive } \\
\text { setting up, etc.) } \\
\text { Question \#5 and 6: landscape metric(s) and } \\
\text { spatial scale(s) }\end{array}$ \\
\hline Study design & $\begin{array}{l}\text { Spatial/temporal/spatial and temporal } \\
\text { comparisons }\end{array}$ \\
\hline Measured outcomes & $\begin{array}{l}\text { Abundance, species richness, Shannon } \\
\text { index, etc. }\end{array}$ \\
\hline Susceptibility to bias & Low/medium \\
\hline
\end{tabular}

coded each question in a different row. The database is freely accessible and easily searchable (Additional file 4).

\section{Data extraction strategy Narrative syntheses}

For all specific questions, we first extracted results into tables. Only the statistically tested results were included. For each species or group of species, we extracted the effects of exposure/intervention and categorized them as positive, negative or neutral. Neutral effects referred to comparison between control and treatment that were statistically non-significant, i.e. there was no difference between the two. One reviewer performed data extraction, and a second reviewer double checked all uncertain cases.

\section{Meta-analyses}

We performed meta-analyses for the specific question \#2 that gathered enough homogeneous (in terms of comparators and outcomes) studies with the required statistics. As specific inclusion criteria for the meta-analysis, in addition to those used for the whole review, primary studies had to report mean, sample size and some measurement of variation for insect abundance or species richness for both LTI and another habitat away from LTI serving as control. In details, insect species richness, abundance and 
its proxies (density, number of burrows/mounds, but not occurrence) were used as response variables in the metaanalyses. In each primary study, for both LTI verges and control sites away from LTIs, we extracted sample sizes, outcome means, and measures of variation (standard deviation, standard error, variance or confidence interval) from tables, text, published raw data (e.g. in appendices), data provided to us by the authors, and graphs (using Get-Data Graph Digitizer 2.26). In addition, when measures of variance were missing, they were either recalculated from raw data, retrieved from statistical test values, or estimated via data imputation [46]. Abundances for either species groups or individual species were extracted. If a study reported the abundances for both a group and its component individual species, we only used the latter. Five studies measured the biodiversity of insects at various distances from LTI verges [47-51]. In these cases, we used values of the furthest distance as controls.

To address our research question, we investigated in meta-analyses a set of candidate moderators (Table 8)

Table 8 Candidate moderators by category

\begin{tabular}{|c|c|c|}
\hline Category & Variable & Description \\
\hline (1) $\mathrm{LTI}$ & LTI type & $\begin{array}{l}\text { LTI category: railway, pipe- } \\
\text { line, power line, waterway, } \\
\text { highway, other roads }\end{array}$ \\
\hline (2) Habitat contrast & Habitat contrast & $\begin{array}{l}\text { Contrast between vegeta- } \\
\text { tion type of LTI verges and } \\
\text { habitats away from LTI. } \\
\text { Coded as low, medium, } \\
\text { high }\end{array}$ \\
\hline \multirow[t]{3}{*}{ (3) Insect } & Trophic level & $\begin{array}{l}\text { Feeding guild of group and } \\
\text { individual species: primary } \\
\text { consumer, pollinator, } \\
\text { secondary consumer, } \\
\text { omnivore }\end{array}$ \\
\hline & Habitat specialization & $\begin{array}{l}\text { Habitat preference of indi- } \\
\text { vidual species: generalist, } \\
\text { specialist of open habitats, } \\
\text { specialist of forest habitats }\end{array}$ \\
\hline & Conservation status & $\begin{array}{l}\text { Category for individual spe- } \\
\text { cies: species of conserva- } \\
\text { tion value, pest/invasive } \\
\text { species, other species }\end{array}$ \\
\hline \multirow[t]{3}{*}{ (4) Study design } & $\begin{array}{l}\text { Type of literature } \\
\text { Replication }\end{array}$ & $\begin{array}{l}\text { Grey versus non-grey } \\
\text { literature } \\
\text { True spatial replication } \\
\text { versus other cases }\end{array}$ \\
\hline & Sample location & $\begin{array}{l}\text { Transparent and systematic } \\
\text { procedure for the selec- } \\
\text { tion of sample plots loca- } \\
\text { tion selection (randomiza- } \\
\text { tion, fixed distances, grids) } \\
\text { versus other cases }\end{array}$ \\
\hline & Study duration & $\begin{array}{l}\text { Number of sampling years: } \\
\text { single year versus more } \\
\text { than } 1 \text { year }\end{array}$ \\
\hline
\end{tabular}

from three broad categories: (1) LTI type; (2) habitat contrast between verges and compared habitats; and (3) attributes of insect species/groups. Finally, we tested moderators related to (4) the design of the studies in order to check for potential biases.

We used five LTI categories (1): railways, pipelines, power lines, waterways, highways and other roads. We discriminated highways from non-highway roads because road width, verge width and disturbances (traffic, noise, light, pollution) can vary considerably between the two types.

In addition to LTI type, we recorded the contrast between LTI verge and the compared habitat as a potential moderator (2). The contrast was considered as low when the vegetation types were roughly similar (e.g. grassy verges compared to grassland, meadow, natural open vegetation), as medium when grassy verges were compared to arable field, and as high when the vegetation types were dissimilar (e.g. grassy verges vs. forest stands).

To evaluate whether heterogeneity in insect response to LTI could be accounted for by species-specific traits (3), we assigned individual species and groups of species to a trophic level (primary consumers-e.g. herbivores, pollinators, secondary consumers-e.g. predators and omnivorous). Scavengers were classified as secondary consumers and insects consuming dead wood and dead plant as primary consumers. In addition, we extracted habitat preference for each individual species of butterflies, bees, bumblebees, ground beetles and ants. We encoded habitat preference in three categories: generalist, specialist of open habitats and specialist of forest habitats. We extracted trophic level and habitat preference information from databases and literature [48, 52-67], and, whenever possible, completed missing information for trophic level with expert knowledge. Finally, we classified individual species according to their conservation status. We based this classification on information given by the authors: we classified threatened, endangered and rare species as species of conservation value; we classified pest, invasive (exotic) species, and species causing infestation as pest/invasive species; in the absence of such information, we classified species as other species.

To test for potential methodological bias, we extracted moderators related to the design of the studies (4) (i.e. type of literature, type of replication, sample plots location and study duration).

\section{Data synthesis and presentation Narrative syntheses}

For each specific question, we produced a narrative table that summarized the key results of relevant studies and we wrote a narrative synthesis that includes descriptive statistics and explanatory text. Whenever possible, we organized the findings from included studies by grouping 
them into categories based on taxonomic group, type of LTI, intervention and/or comparator.

\section{Meta-analyses}

We used the Hedge's d standardized mean difference as a measure of the effect size for both species richness and abundance [68]:

$$
d=\frac{\overline{\mathrm{X}}_{G 2}-\overline{\mathrm{X}}_{G 1}}{S_{\text {pooled }}} J
$$

where $\overline{\mathrm{X}}_{\mathrm{G} 1}$ and $\overline{\mathrm{X}}_{\mathrm{G} 2}$ were the means of group 1 (control sites away from LTIs), and group 2 (treatment sites on LTI verges) respectively. Thus, the effect size $d$ was positive if the species richness or abundance was higher in LTI verges than in habitats away from LTIs.

$S_{\text {pooled }}$ was the pooled standard deviation of the two groups:

$$
S_{\text {pooled }}=\sqrt{\frac{\left(n_{G 2}-1\right) S D_{G 2}^{2}+\left(n_{G 1}-1\right) S D_{G 1}^{2}}{n_{G 1}+n_{G 2}-2}}
$$

where $\mathrm{SD}=$ standard deviation, and $\mathrm{n}_{\mathrm{G} 1}$ and $\mathrm{n}_{\mathrm{G} 2}$ were the sample sizes of group 1 and group 2.

$\mathrm{J}$ was a correction term that mitigated small sample size bias.

$$
J=\left[1-\frac{3}{4\left(n_{G 1}+n_{G 2}-2\right)-1}\right]
$$

The variance for Hedge's d can be calculated with:

$$
v a r=\frac{n_{G 1}+n_{G 1}}{n_{G 1} n_{G 2}}+\frac{d^{2}}{2\left(n_{G 1}+n_{G 2}\right)}
$$

If within a study, the cases were not independent (e.g. several treatments were compared to the same control), an overall corrected sample size $\mathrm{N}_{\text {corrected }}$ was calculated for the non-independent cases as the effective sample size on the field, therefore counting the sample size of the control only once, following the method described in Gleser and Olkin [69]:

$$
N_{\text {corrected }}=n_{G 1}+\sum_{1}^{i} n_{G 2 i}
$$

With $\mathrm{n}_{\mathrm{G} 1}$ the sample size of the control and $\mathrm{n}_{\mathrm{G} 2 \mathrm{i}}$ the sample sizes of each treatment compared to the same control.

$S_{\text {pooled }}$ J, Hedge's $d$ and the variances for Hedge's $d$ for those cases were corrected using $\mathrm{N}_{\text {corrected }}$ instead of the sum of $\mathrm{n}_{\mathrm{G} 1}$ and $\mathrm{n}_{\mathrm{G} 2}$ :

$$
S_{\text {pooled.corrected }}=\sqrt{\frac{\left(n_{G 2}-1\right) S D_{G 2}^{2}+\left(n_{G 1}-1\right) S D_{G 1}^{2}}{N_{\text {corrected }}-2}}
$$

$$
\begin{aligned}
& J_{\text {corrected }}=\left[1-\frac{3}{4\left(N_{\text {corrected }}-2\right)-1}\right] \\
& v_{\text {corrected }}=\frac{1}{n_{G 1}}+\frac{1}{n_{G 2}}+\frac{d^{2}}{2\left(N_{\text {corrected }}\right)}
\end{aligned}
$$

A matrix of variance-covariance of the effect-size estimates was calculated and used as the variance components in the subsequent models [69].

The statistical significance of the moderator effects was tested using mixed models with the restricted maximum-likelihood estimator (REML). We nested the study cases within the studies as random effects for each analysis, which allowed effect size estimates to vary not only owing to sampling error, but also owing to biological or environmental differences between organisms and studies. A study case referred to an individual effect size extracted from a study (e.g. abundance of a particular insect species); a given study possibly gathering multiple study cases (e.g. abundance of several insect species).

Under a mixed model, the weight assigned to each effect size $\left(w^{*}\right)$ was set as the inverse of the sum of two variance components $w^{*}=1 /\left(w+\tau^{2}\right)$, where $w\left(=1 / \mathrm{se}^{2}\right)$ was the unique sampling variance for each study (withinstudy error) and $\tau^{2}$ was the estimated pooled variance of the true effects across all studies (between-studies variance).

In a first step, we computed the grand mean effect size for each response variable (i.e. abundance and species richness) by combining data from all LTIs and species. Additionally, the data sets were analyzed for publication biases using funnel plots, plots of the mean cumulative meta-analysis by publication year (Additional file 8), and the fail-safe number modified by Rosenberg [70]. At this stage, we also tested the effects of the design of studies.

Then, for testing the effects of the moderators (type of LTI, habitat contrast between verge and compared habitat, attributes of insect species/groups, Table 8), we avoided problems associated with confounding factors by constructing independent subsets of data in a hierarchical approach (see Fig. 7). We restricted our analyses to categorical moderators that ensure at least two studies in each resulting categories. Thus, in some cases, we either combined similar categories to increase the sample size or deleted the categories that did not meet the sample size criteria. In particular, we combined pipelines and power lines to overcome small sample sizes, and because these two LTIs have similar verges (i.e. strips of grass or shrub under power lines and above buried pipelines).

Concerning the abundance, we tested the "habitat specialization" effect comparing models with and without 
interaction terms with AIC and Anova. We did not use the AIC corrected for small sample size (AICc) because the sample sizes were large enough in regards to the numbers of parameters of the models. We tested (1) the interaction between habitat specialization and trophic level for the subset of data on highways (the only LTI with enough data), and (2) the interaction between habitat specialization and LTI type for the subset of data on pollinators (the only trophic level with enough data). The "forest specialist" category was discarded as it only gathered five cases. Given the absence of statistically significant interaction in both cases (the AIC of the model with interaction was not lower by 2 points or more than the AIC of the model without interaction), the "habitat specialization" factor was not taken into account in further meta-analyses. We pooled all species irrespective of their habitat specialization for subsequent analyses.

We evaluated the effect of the trophic level on the abundance of insects for each LTI type separately (see Fig. 7). Despite the large number of cases for abundance $(\mathrm{N}=661)$, their distribution between moderator categories prevented us to run a model using the conservation status of each species as moderator $(\mathrm{N}$ pest/invasive $=2$, see Additional file 7). In a last step, we checked if contrast between verges and compared habitats did not affect our results by testing the interaction between habitat contrast (low/medium vs. high) and trophic level for the subset of data on highways (the only LTI with enough data).

For species richness, the uneven distribution of effect sizes and their small number $(\mathrm{N}=48)$ prevented us to evaluate the effect of trophic level. We were thus unable to move further beyond evaluating the effect of LTI type.

For all data analyses, total heterogeneity, $\mathrm{Q}_{\mathrm{T}}$, was partitioned into heterogeneity explained by the model, $\mathrm{Q}_{\mathrm{M}}$, and heterogeneity not explained by the model, $Q_{E}$ (i.e. $\mathrm{Q}_{T}=\mathrm{Q}_{\mathrm{M}}+\mathrm{Q}_{\mathrm{E}}$ ). The statistical significance of $\mathrm{Q}_{\mathrm{M}}$ and $\mathrm{Q}_{\mathrm{E}}$ were tested against a $X^{2}$ distribution.

Because some case studies may not be fully independent as they came from the same primary study, we performed a sensitivity analysis to assess the robustness of the main results (Additional file 9).

Meta-analyses were conducted in R 3.3.2 [45] using the metafor R package [71].

\section{Results}

\section{Descriptive statistics of the systematic review Searches, screening and quality assessment}

We performed the searches in the Web of Science Core Collection publication database, Zoological Records publication database and in Google Scholar on April 27nd 2015, February 1st 2016 and March 4th to 9th 2016 respectively. For homogeneity of the year of publication between the two publication databases, we did not include the articles issued from Zoological Records released in $2016(\mathrm{~N}=5)$. We performed the call for grey literature on April 21nd, 2015.

Details on search results and outcomes of the screening and quality assessment processes are summarized in Fig. 1.

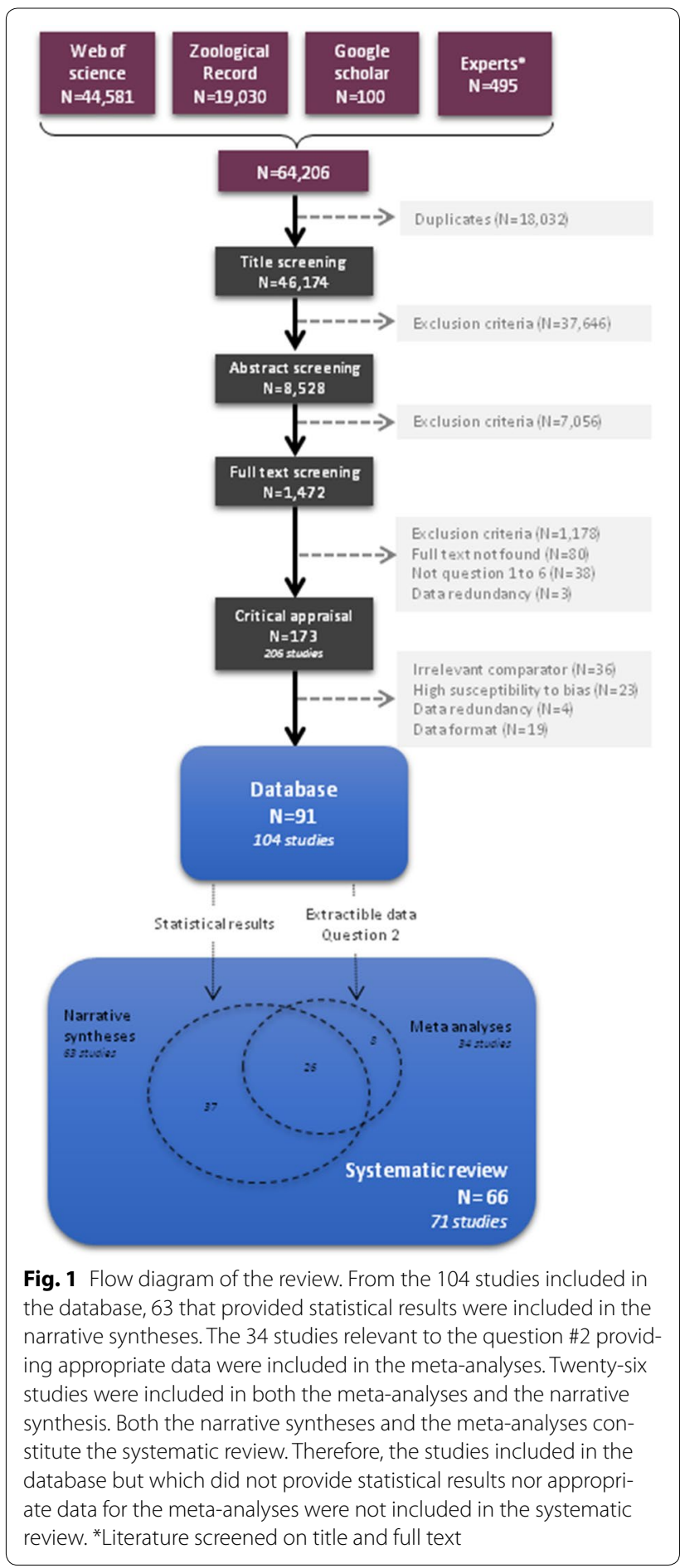


Searches based on title, abstract and keywords with English search terms returned a total of 44,581 articles in the Web of Science Core Collection publication database, and 19,030 articles in Zoological Records publication database. Of the test list of 102 key articles identified by subject experts, 84 were indexed in the Web of Science Core Collection and 83 were found with our search strings combination; 51 were indexed in the Zoological Records and 49 were found with the search strings. By combining both Web of Science Core Collection and Zoological Records searches, 8 articles published in non-indexed journals out of the 102 articles of the test list were not found (see Additional file 2). The search on Google Scholar and the call for grey literature returned 100 and 495 documents respectively, the 495 grey literature documents originating from 52 persons.

The removal of duplicates led to 46,174 documents from which 8528 articles remained after title screening. Of them, 1472 articles were included after abstract screening of which we were unable to retrieve 80 fulltexts, leaving 1392 full-texts to screen. At full text screening, the most common reasons for exclusions were: non-temperate climate, incorrect type of study (reviews, modeling studies and studies making policy recommendation without making experimentations), and language (e.g. title and abstract in English, but with whole-text in German). During the full text screening, 38 articles were relevant to our broad question but answered other specific synthesis questions than the six listed in Table 1 , and thus excluded from subsequent steps. After full-text screening 173 articles remained.

These 173 articles contained reports on 206 studies that were critically appraised individually (an article was split into different studies when the article dealt with more than one of our specific questions). Critical appraisal furthermore excluded 102 studies: 4 studies because of data redundancies, 42 studies that had irrelevant comparator(s) for the purpose of our review, 29 studies because of an inappropriate data format (i.e. studies that did not allow separating relevant results from irrelevant ones -exposures/population not under review scope), and 27 studies with high susceptibility to bias. The main reasons for high susceptibility to bias were major confounding factors, unclear sampling design and/or protocol, and the absence of any replication.

Figure 2 shows the number of studies with low, medium and high susceptibility to bias for each specific question.

After critical appraisal, 91 articles remained. They constituted 104 studies listed in Additional file 4.

Additional file 5 lists the studies which were rejected on full-text assessment and critical appraisal, together with the reasons for exclusion. This file also contains the list of articles that we did not find in full-text.

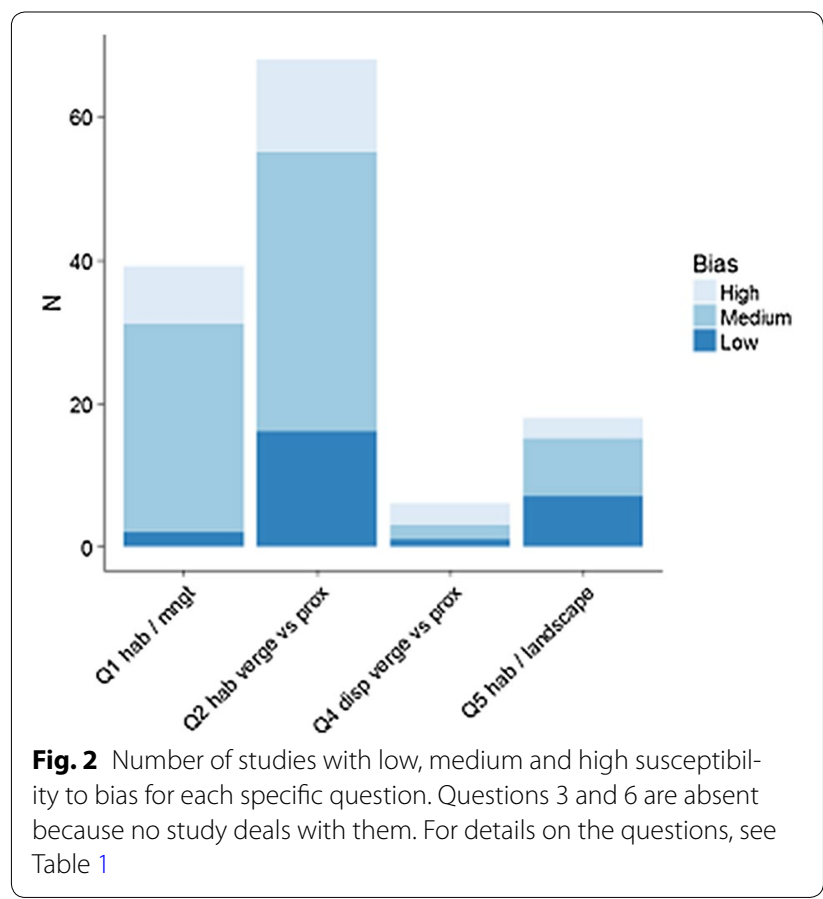

\section{Narrative synthesis of included studies}

Among the 91 included articles, 84 came from the searches in scientific publication databases (WOS CC and ZR). The grey literature call provided only 6 other documents and one article was only retrieved on Google Scholar. Ten of the articles were in French, the other ones in English. The most common type of document was scientific journal articles $(\mathrm{N}=84), 4$ Ph.D. chapters, 2 technical reports and one MSc report were also included.

Among the 104 included studies (Additional file 4), 31 studies evaluated the effect of verge management on insect biodiversity (question \#1) and 55 studies compared insect biodiversity in verges to the one of other habitats (question \#2). No study dealt with the influence of verge management on insect dispersal (question \#4), neither with the influence of surrounding landscape on dispersal along the verges (question \#6). Three studies compared insect dispersal along verges with insect dispersal in habitats away from LTIs (question \#3). Fifteen studies focused on the influence of the surrounding landscape on the biodiversity hosted by verges (question \#5).

Most of the studies were conducted in Europe $(\mathrm{N}=62)$, particularly in France, United Kingdom, Poland and Netherlands (Figs. 3, 4), 20 studies took place in North America (all of them in the United States of America), 10 studies were conducted in Australia-Oceania, while the other continents were poorly represented (Asia $\mathrm{N}=6$, Africa $\mathrm{N}=5$ and South America $\mathrm{N}=1$ ).

The language criteria (English or French) used during screenings and the dominant nationality of the panel of 

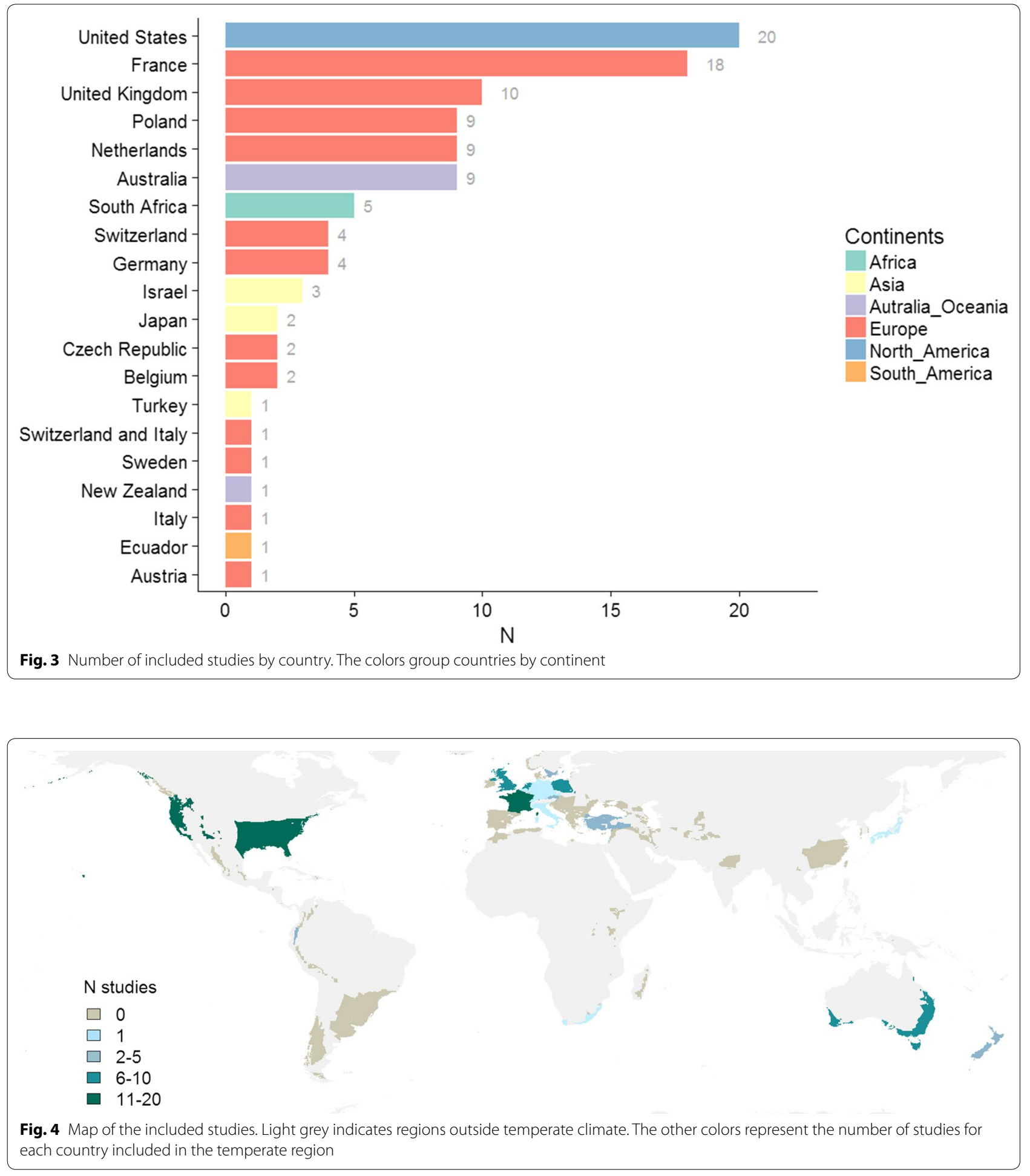

experts contacted can explain the high number of studies conducted in France. Indeed, 8 of the 18 studies conducted in France belonged to documents written in French, and we collected 7 of these 8 studies through our call for grey literature.
The oldest retrieved study included in this review was published in 1972. Altogether a global increase in the number of studies can be observed along the decades (Fig. 5a).

Roadside verges were by far the most studied type $(\mathrm{N}=65)$, followed by waterway banks $(\mathrm{N}=30)$, railway 

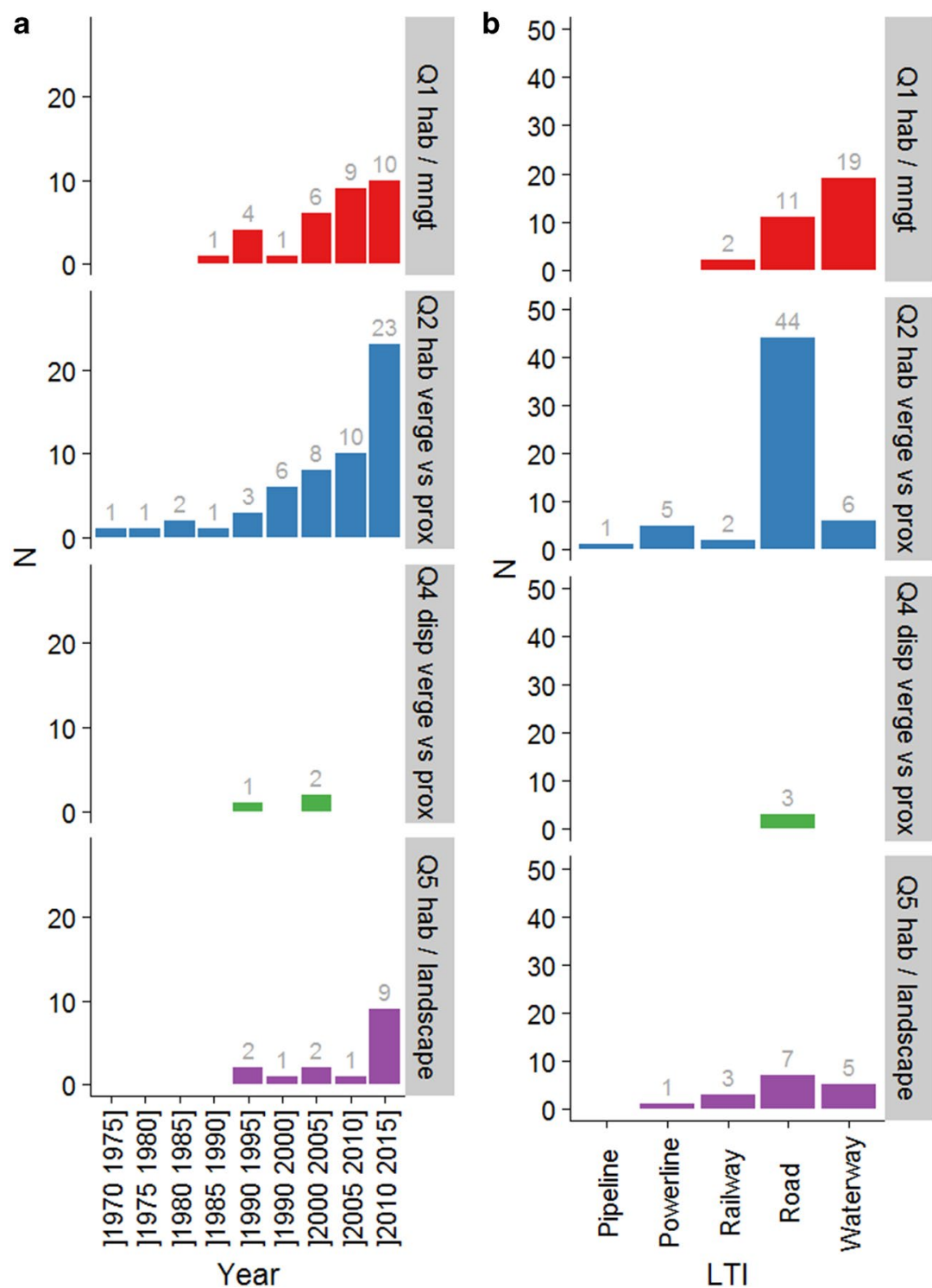

Fig. 5 Year of publication of the studies that addressed our six specific questions (a), and LTIs under study for our specific questions (b). In $\mathbf{b}$, the sum exceeds the number of studies included in the database because one study can imply more than one type of LTI. Questions 3 and 6 are absent because no study dealt with them. For the details on the questions Q1 to 6, see Table 1

embankments $(\mathrm{N}=7)$, power line $(\mathrm{N}=6)$ and pipeline verges $(\mathrm{N}=1)$. The specific question that compares insect biodiversity along and away from LTI verges focused disproportionately on roads (44 among 58 studies, question \#2 Fig. 5b), and the only studies about insect dispersal focused on road verges. On the contrary, waterway banks gathered more than half studies about the effect of management practices on insect biodiversity (19 among 32 studies, question \#1 Fig. 5b).

Coleoptera and butterflies were the most studied taxa ( $\mathrm{N}=47$, Fig. 6). Sixteen studies focused on composite groups: on one side, studies on invertebrates or arthropods that include insects, and on the other side, studies on insects without precision about which insects the study dealt with. Ants were investigated by 13 studies and bees and/or bumblebees by 10 studies. The other taxa were, among others, Odonata, Diptera, Hemiptera, Orthoptera and Heteroptera.

Among the outcomes, more than 3/4 of the studies used measures related to abundance (abundance, density, occurrence, frequency, patch occupancy...), of group or individual species. Nearly $2 / 3$ of the studies presented indices of species richness (species richness, Shannon, Simpson, Hill, evenness index, Piélou equitability...). Nearly $1 / 3$ of the studies used indices of similarity or dissimilarity between communities (Jaccard, Sørensen, 


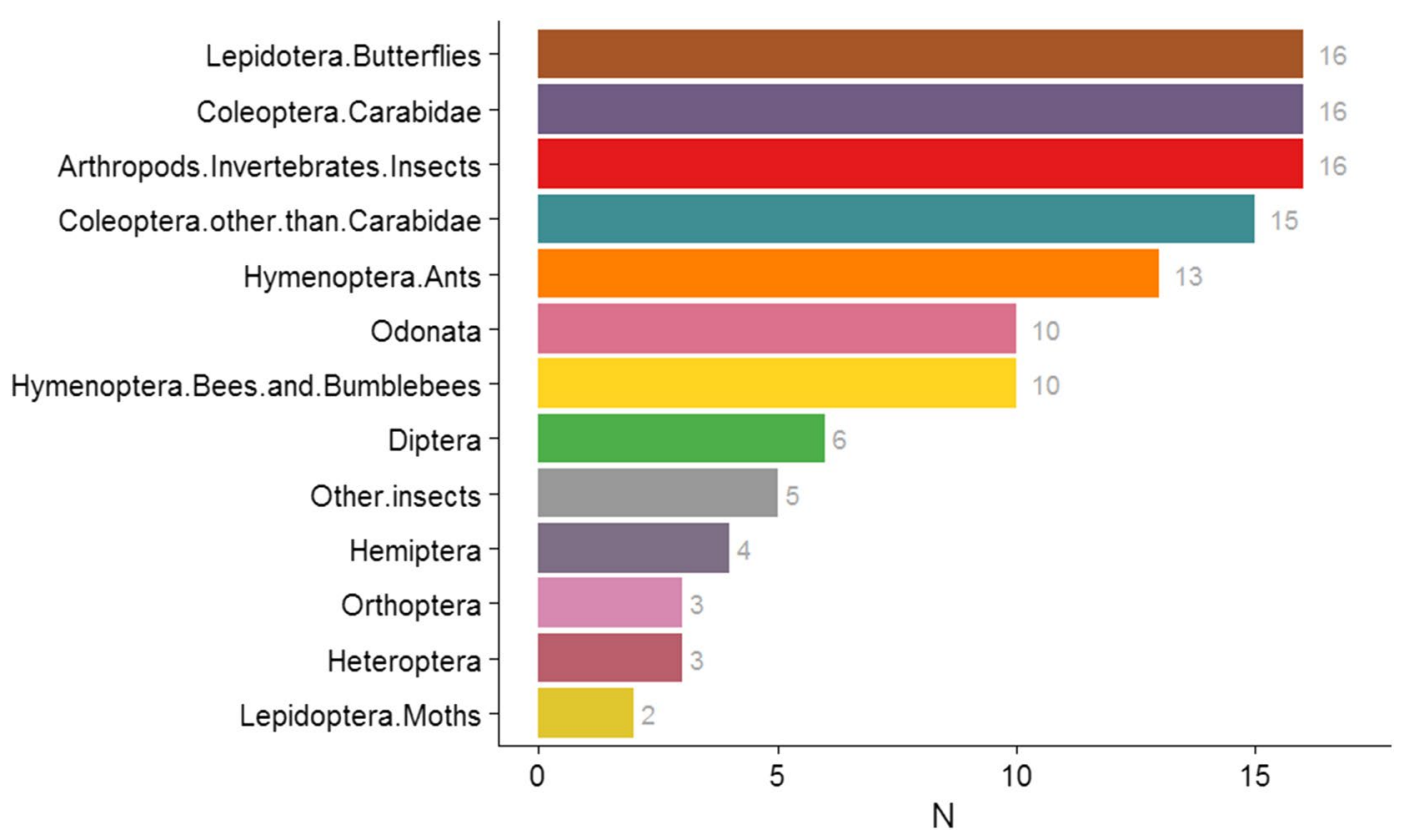

Fig. 6 Taxonomic insect groups of the included studies. The sum exceeds the number of included studies because some studies included more than one taxonomic group

Renkonen indices, Bray-Curtis dissimilarity...), and the same proportion focused on community composition (species traits, feeding guilds, relative abundance...).

Of the 104 included studies, 101 had a control/intervention or control/exposure design, two studies had a BACI design and the remaining one had a before/after design.

Details about the studies (language, specific question addressed, country, biological group, LTI, study design, outcomes, susceptibility to bias etc.) are available in Additional file 4.

\section{Data synthesis and presentation}

For each specific question, we first began with a narrative synthesis of the studies and summarized key results in a table. The lack of studies, or the broad variation in the type of investigations conducted, prevented us from using formal meta-analytical techniques for the questions $3,4,6$ and 1,5 respectively.

For the specific question gathering the most retained articles, i.e. question \#2 (Habitat in LTI verges vs. at proximity), both narrative synthesis and meta-analyses were conducted. The narrative synthesis provides the context and background for the quantitative meta-analyses. In addition, some of the articles, which did not provide suitable data to be included in the meta-analyses, presented results that can be included in a narrative synthesis. The meta-analyses especially focus on LTI types and species traits (trophic level and habitat preference), and go deeper than the narrative synthesis on these aspects. Thus, the narrative synthesis and the meta-analyses are complementary, both providing a different perspective.

\section{Narrative syntheses}

The narrative syntheses of secondary review questions were based on 63 studies out of the 104 included studies. We put aside the studies (indicated in Additional file 4) that met our inclusion criteria but that did not provide statistical results to compare control and treatment. The qualitative tables synthesizing key results are provided in Additional file 6.

\section{Question \#1: Do LTI verge management practices increase, decrease or have no effect on insect biodiversity?}

Seventeen articles included in our systematic review provided statistical results relevant to this specific question. Their key results are provided in Additional file 6: Table A1.

Among the different types of verge managements,

- 6 articles (group a in Additional file 6: Table A1) dealt with the modification of vegetation composition: alien plant management, plantation, sowing [72-77].

- 5 articles (group b) studied the influence of physical modifications: type of embankment, channelization, pavement, type of bottom [78-82].

- 6 articles (group c) concerned mowing and/or grazing [83-88]. 
Management of verges aiming at restoring vegetation seemed to have positive to neutral effects on insect biodiversity.

Indeed, the modification of vegetation composition to restore "initial" or "natural" vegetation had no effect on the abundance of aquatic insects [72], on the composition of ant communities [73], and on the abundance of Apis mellifera [74]. Similarly, the modification of vegetation did not affect the abundance of 9 Odonata species [75], and the ground beetle biodiversity indices [76].

Nevertheless, modifications of vegetation composition aiming at getting rid of non-native species promoted bee species richness and abundance [74], the species richness, abundance, taxonomic distinctiveness of Odonata and the abundance of 13 Odonata species [75], as well as the abundance of aquatic insects [77]. The only contrasting result referred to the abundance of Chlorolestes umbratus, a rare Odonata species, which was lower along restored riparian corridors when compared to sites invaded by alien trees [75].

Human impact (artificialization) on the LTIs (mineral and synthetic embankment, channelization and pavement) appeared to have mainly negative to neutral effects on the biodiversity of the verges.

The pavement of railway verges lowered Orthoptera biodiversity (in comparison with unpaved verges [81]), and the use of synthetic bottom in stormwater retention ponds (dig near highways to retain stormwater runoff and pollutants) lowered the richness of Odonata species (in comparison with natural bottom [82]). Moreover, Cavaillé et al. [78] observed a lower number of Coleoptera in artificial riverbank embankments as compared to vegetated one, similar to the observation of Paetzold et al. [80] that channelization lowered the abundance and species richness of rove beetles in riverbanks with hydropeaking flow regime. On the contrary, Dymitryszyn [79] found no difference in carabid abundance and diversity before and after roadside reconstruction (replacement of the substratum and partial paving of the shoulders), like Paetzold et al. [80] who did not observe difference in ground beetle' species richness and abundance between channeled and unchanged riverbanks. Similarly, Ruspolia nitidula abundance (Orthoptera) appeared similar between paved and unpaved railway verges [81], and Cavaillé et al. [78] found similar numbers of Coleoptera species in vegetal and mixed (vegetal and mineral) river embankments. Only 2 studies [78, 80] showed a positive influence of artificialization of verges on some insects: the frequency of exotic Coleoptera species appeared higher for mixed as well as for mineral riverbanks when compared to vegetal riverbanks [78]. Similarly, Paetzold et al. [80] showed that channelization increased the abundance and species richness of rove beetles in riverbanks with a natural flow regime.
The currently available results about the influence of mowing intensity were inconsistent among taxonomic groups. Ward and Mill [87] observed that disturbance by cattle reduced the occurrence of Calopteryx splendens (Odonata). Higher mowing frequency resulted in higher numbers of butterfly roadkills and the occurrence of blackspot sites [86], while no effect of mowing was observed for ants [85]. The 3 remaining studies $[83,84,88]$ compared practices that combined various parameters of mowing management (frequency, period, removal or not of hay, and leaving of unmown parts or not), so that the independent effect of each parameter was difficult to extract. Overall, many comparisons of management regimes were not statistically significant. In Noordijk et al. [83], hay removal did not affect insect abundance and flower visits in roadsides, similarly to their results published in 2010 [84] where no effect was detected on species richness of ground beetles and ants. However, the authors also found that hay removal sometimes increased the species richness of weevils. Mowing frequency did not consistently influence the species richness of weevils [84]. Noordijk et al. [84] showed that mowing once a year favored ant species richness in comparison to non-mowing and mowing twice a year, whereas they showed in their 2009 and 2010 studies that ground beetle species richness, insect abundance and flower visits were higher in roadsides mown twice a year than in roadsides non-mown or mown once a year. Finally, Wynhoff et al. [88] observed that partial mowing of road verges was beneficial for ant abundance, especially Lasius flavus and Lasius niger, while late mowing favored ant abundance of some other species, especially Myrmica rubra.

\section{Question \#2: Is the biodiversity of LTI verges equal to, higher,} or lower than the biodiversity of habitats away from the LTI?

The key results of the studies included in the narrative synthesis are provided in Additional file 6: Table A2.

Among the 37 studies with relevant statistics that compared the biodiversity hosted by LTI verges to the biodiversity of habitats away from the LTIs, we distinguished 5 main categories:

- Coleoptera (mostly Carabidae) along terrestrial LTIs (group d) [48, 66, 67, 79, 89-94].

- pollinators (bees, bumblebees, hoverflies and butterflies) along terrestrial LTIs (group e) [31, 74, 95-100].

- "unwanted species" (invasive species, pests...) along roads (group f) [101-107].

- other taxa along terrestrial LTIs (group g) [49, 67, 90, 91, 100, 108-113].

- various taxa along waterways (group h) [114-116]. 
In most of the articles (28 among 37), the habitats on the LTI verges and away from the LTIs were rather similar (e.g. comparison of herbaceous road verges with grasslands). In one paper, the compared habitat greatly differed from the LTI verge: Silverman et al. [94] compared an open deforested pipeline corridor to the intact adjacent forest representing the initial habitat before the pipeline construction. Eight articles compared the LTI verges with several types of habitats in each study, both similar and dissimilar [48, 79, 89, 92, 96, 98, 101, 115], sometimes relating the results with species habitat or feeding preferences $[48,79,115]$. In the meta-analyses, we tested whether this heterogeneity between comparisons influenced the results (see "Meta-analyses" section).

Detecting trends on the potential of terrestrial LTI verges as habitats for Coleoptera was challenging. Most of the comparisons made between LTI verges and other habitats were statistically non-significant $[48,66,67,79$, 90-92]. Depending on the indices considered, LTI verges hosted higher, similar or lower insect biodiversity than other habitats [93], and the results for individual species abundances were also diverse [91, 94]. The differences for Coleoptera between the biodiversity of verges and the one of the habitats away from LTIs depended on the nature of the habitats compared $[48,66,79,92]$, and sounded congruent with the habitat preference classification used by the authors $[48,66]$. Indeed, Knapp et al. [48] showed that the species richness of forest specialist ground beetles and large rove beetles was lower on roadsides than in forest habitats, whereas the species richness of open specialists was higher on roadsides than in forest habitats. Similarly, the species richness of ground beetles stenotopic for heathland/drift sand areas was statistically lower in roadside verges than in heathland nature reserves [66].

Globally, terrestrial LTI verges seemed to host similar to richer pollinator communities than habitats away from LTIs.

Indeed, no difference was found (i) for pollinators (of several groups) in roadsides and power line corridors (in comparison with forests and forest edges [96]), (ii) for hoverflies along railway embankments (in comparison with grasslands [31]), and (iii) for butterflies in highway verges (in comparison with controls not detailed [100]). LTI verges sometimes hosted more abundant and diverse pollinator communities than habitats outside verges. Indeed, Hanley and Wilkins [97] showed a higher bumblebee abundance along road verges than in adjacent (unplowed) field margins. Similarly, Moroń et al. [31] outlined higher abundance and species richness of bees and butterflies along railway embankments than in grasslands, close to the observations of Russell et al. [99] who found more bees in power line corridors than in grasslands.

Finally, some studies showed more complex responses of pollinators. Osgathorpe et al. [98] found that bee abundance differed between years; in Bailey [95] abundance of bees and bumblebees differed between periods (over the same year) and species. In addition, in Mallard [50], the results are inconsistent between sites: butterfly species richness and abundance were higher along verges than in forests in some sites, and lower in others.

Mainly, road verges appeared to host comparable to greater abundance of "unwanted species" than habitats away from roads. Indeed, proxies of abundance of "unwanted species" between road verges and other habitats were not different for mosquitoes [101], which was consistent with the results for the invasive red fire ant Solenopsis invicta in Texas (as compared with grasslands [105]), and for the monarch butterfly parasite Lespesia archippivora (in comparison with prairies [107]). On the other side, Braun and Flückiger [102] recorded more abundant apple aphids Aphis pomi on road verges than in control sites, which tallied the results of Loch and Zalucki [104] for the pink wax scale Ceroplastes rubens (when compared to gardens), and that of Vogt [106] for the imported red fire ant Solenopsis invicta in Mississippi (in comparison with similar habitats in vicinity).

One exception was the study by Kline [103], who reported that the abundance of Culicoides ssp. larvae was lower in road ditches than in adjacent salt marshes.

Finally, (non-pest) ants, (non-pest) aphids, Odonata and Heteroptera were the last other groups covered by the comparison of insect biodiversity between terrestrial LTI verges and other habitats. We also included in this category the results from studies that only used generic terms such as "insects".

These taxonomic groups showed mostly similar to higher biodiversity on terrestrial LTI verges than in other habitats.

All studies except Ditsworth et al. [108], Freitag et al. [109] and Wilkaniec et al. [113] reported no statistically significant differences between the biodiversity of verges and those of the compared similar habitats for at least one of their comparisons. However, Ditsworth et al. [108] reported that a power line corridor hosted more individuals of Cicadellidae, Aphididae, Lepidoptera and Thysanoptera than pynion-juniperus woodland.

Three studies agreed on higher abundance of ants in road verges than in similar habitats away from LTIs: Freitag et al. [109] for Formica pratensis, Itzhak [110] for Messor semirufus, Tapinoma israele and of T. erraticum, and Major et al. [91] for 3 ant morphospecies. Le Viol et al. [67] also indicated that 2 Odonata and 3 
Heteroptera families were more abundant in man-made highway stormwater retention ponds than in natural ponds. Martinez and Wool [112] evidenced that aphids inducing galls on Pistacia trees were mainly more abundant on Pistacia trees of roadsides than on Pistacia trees in the shrub land, and interpreted this fact with the higher water supply along roads, which favors both tree and aphid growth. Similarly, Wilkaniec et al. [113] calculated a Shannon-Weaver index of aphids higher in roadsides than in the shrub habitats.

In very few studies, the abundance of insects was lower in verges than in habitats away from LTIs. In fact, Le Viol et al. [67] documented one Odonata and 2 Heteroptera families less abundant in highway retention ponds than in natural ponds, and Ditsworth et al. [108] reported that the abundance of Coccoidea (Hemiptera) was lower below a power line than in pynion-juniperus woodland.

We were not able to draw any general pattern from the last 3 articles that compared the insects in riverbanks and in other habitats, as the conclusions depended i) on the taxonomic groups: Schipper et al. [116] found higher abundance of Coleoptera and Diptera in floodplain but no difference for the other taxonomic groups tested (Caelifera, Heteroptera, Homoptera, Hymenoptera, Lepidoptera and Siphonaptera); ii) on the biodiversity indices considered: Houston et al. [115] outlined higher species richness but lower abundance of termites in riparian forest than in pasture.

To summarize, the insect biodiversity hosted by the LTI verges seems mostly similar to the one supported by habitats away from LTIs. In a number of cases, LTI verges may support richer communities. The meta-analyses that follow enlighten the causes of the inconsistencies.

\section{Question \#3: Do LTI verge management practices increase, decrease or have no effect on insect dispersal?}

No study evaluated the influence of verge management on insect dispersal.

\section{Question \#4: Is insect dispersal on LTI verges equal to, higher, or lower than to their dispersal in habitats away from the LTIs?}

There were very few studies dealing with the dispersal of insects along LTI verges.

Only 2 articles provided relevant statistical results to compare dispersal along the verge to dispersal measured in another habitat. Their key results are provided in Additional file 6: Table A3.

Brunzel et al. [117] compared the colonization events of a moth species in sites linked or not with the nearest population by a road and noticed that the presence of a road increased the probability of colonization of Tyria jacobaeae. Vermeulen [118] evaluated carabid dispersal thanks to the mark release recapture method and found that the rate of movements of Pterostichus lepidus, a wingless species specialist of open habitat, was lower along road verges than in the adjacent open areas.

To summarize, given the lack of evidence on the insect dispersal along LTI verges, we cannot conclude on the influence of verge management on dispersal and on the potentialities of LTI verges as corridors for insect dispersal.

\section{Question \#5: Is the insect biodiversity of LTI verges dependent on the surrounding landscape?}

Nine articles included in our systematic review provided us with relevant statistical results to answer this specific question. Their key results are provided in Additional file 6: Table A4.

Among them,

- 7 articles studied the influence of urbanization (group i).

- 5 articles included agriculture in the surroundings (group j).

- 4 articles evaluated the effect of protected areas and (semi-)natural habitats in the vicinity (group k).

- 3 articles included the proportion of forested areas (group l).

The spatial scale studied, which can explain the variability between results, varied considerably among articles. It ranged from the land use directly adjacent to the LTI verge [119], to land uses in radii of a few hundred meters [81, 120], up to the global catchment scale [121]. The landscape metrics were generally continuous (e.g. proportion of urban area), but 3 studies were based on the comparison of sites classified according to their surrounding landscape (e.g. rural-natural versus urban landscape) $[119,122,123]$.

It seemed that urbanization in the surrounding landscape has negative to neutral effects on the insect biodiversity hosted by LTI verges. Indeed, Dallimer et al. [120], Scher and Thièry [82], Verboven et al. [123], Tagwireyi and Sullivan [122] and Penone et al. [81] all converged toward the fact that surrounding urbanization decreased the species richness of verges for butterflies and Odonata, and both species richness and abundance for bees, hoverflies, ants and Orthoptera. Similarly, Verboven et al. [123] noticed a lower Shannon index of hoverflies in urban sites, while Penone et al. [81] showed that the Community Specialization Index of Orthoptera declined with the rise of urban surfaces. Verboven et al. [123] reported that hoverfly communities were more similar (Sorensen index) between verges surrounded by urban sites than between verges surrounded by rural-agricultural sites. 
Nevertheless, more than half of the comparisons of urban sites with both rural-agricultural and rural-natural sites were statistically non-significant for bee and hoverfly biodiversity indices (abundance, species richness, and Shannon index [123]). Lastly, Dallimer et al. [120] showed multi-faceted and non-linear trends: the effect of the distance to the city center on species richness of butterflies was inconsistent between sites, and species richness of butterflies peaked at intermediate densities of human population.

Agricultural development-another type of human pressure-also seemed to have negative to neutral effects on insect biodiversity in LTI verges. Indeed, in comparison with rural-natural sites, rural-agricultural sites hosted lower bee species richness and Shannon index [123]. Similarly, Magierowski et al. [121] reported a decrease of the ETP (Ephemeroptera-TrichopteraPlecoptera) richness and \% ETP along with the increase of the proportion of crops and grazing areas at the catchment scale. On the other hand, when evaluated at the local scale, the proportion of crops and grazing areas had no visible effect on \% ETP [121]. Bailey [95] could not detect any effect of the proportion of oilseed rape fields on the abundance of individual bee species. In addition, many comparisons of rural-agricultural sites with ruralnatural sites [123] were statistically non-significant for bees (abundance, Sorensen index) and hoverflies (abundance, species richness, Shannon, Sorensen indices). Lastly, Tagwireyi and Sullivan [122] did not find dissimilarity between ant communities in riverbanks in agricultural reaches and those in urban reaches.

The proximity and the proportion of natural habitats or protected areas in the surroundings appeared to have mainly neutral to positive effects. Indeed, ETP richness and \% ETP increased with the proportion of protected areas at the catchment scale, whereas they had no effect on \% ETP at the local scale [121]. Moron et al. [31], observed that the species richness of hoverfly in the verges increased with the increase of the proportion of extensive grasslands in the surrounding landscape while the abundance of bees declined. As previously stated, some bee and hoverfly indices were higher in rural-natural sites than in urban or rural-agricultural sites, even if many comparisons were statistically non-significant [123]. The abundance, species richness and ShannonWeaver diversity index of butterflies did not statistically differ between road verges adjacent to intensive agricultural or urban areas and those adjacent to semi-natural biotopes [119].

Forested areas in surroundings showed mainly neutral to positive effects on insects of the LTI verges. Indeed, Moron et al. [31] evidenced that the species richness of bees, hoverflies and butterflies increased with the proportion of woodland whereas the abundance of bees decreased and the abundance of hoverflies and butterflies were not affected. ETP richness and \% ETP increased with the proportion of forests at the catchment scale, while they had no effect on \% ETP at the local scale [121].

\section{Question \#6: Is insect dispersal on LTI verges dependent on the surrounding landscape?}

No study evaluated the influence of the surrounding landscape on insect dispersal in LTI verges.

\section{Meta-analyses \\ Description of the data}

Quantitative data were extracted from 34 studies among the 55 included in the database for question \#2 (Habitat in LTI verges vs. at proximity). Five studies that appeared in the narrative synthesis were not included in the metaanalyses because the statistical information provided by authors was not sufficient to calculate effect sizes (see details of these studies in Additional file 4). Conversely, 8 studies that did not appear in the narrative synthesis (because of missing statistical tests) were included in the meta-analyses as the available data allowed us to calculate effect sizes (Additional file 4).

Among the 709 study cases extracted, 48 cases in 20 studies referred to species richness (data provided in Additional file 7) and 661 cases in 29 studies referred to species or group abundance (Additional file 7). We estimated the variance of cases with data imputation (i.e. filling this missing variance by using the available data from the other studies) for less than $10 \%$ of the total data $(\mathrm{N}=59$ out of 709$)$.

Most of the study cases were conducted along roads (highways: $47 \%$, non-highway roads: $12 \%$ ), power lines (26\%) and railways (11\%), few of them were on waterways (5\%) or pipelines (1\%). Two-thirds of the cases were from Europe, the others were equally distributed between North America (all in the United States of America) and Australia. Africa and Asia represented less than $1 \%$ of the data.

Among these 709 cases, 41\% concerned Hymenoptera, 20\% Lepidoptera, 19\% Coleoptera and 13\% Hemiptera. Table 9 reports the distribution of sample sizes (abundance and species richness) among LTI type, trophic guild and habitat specialization.

\section{Global meta-analyses and publication bias}

The overall grand mean effect size for abundance was statistically different from zero: insects were more abundant along verges than in habitats away from LTIs $(d=0.174$, $95 \%$ CI $0.000,0.348, \mathrm{p}=0.050$ ). There was a statistically significant heterogeneity in the effect sizes $(Q=2303.16$, 


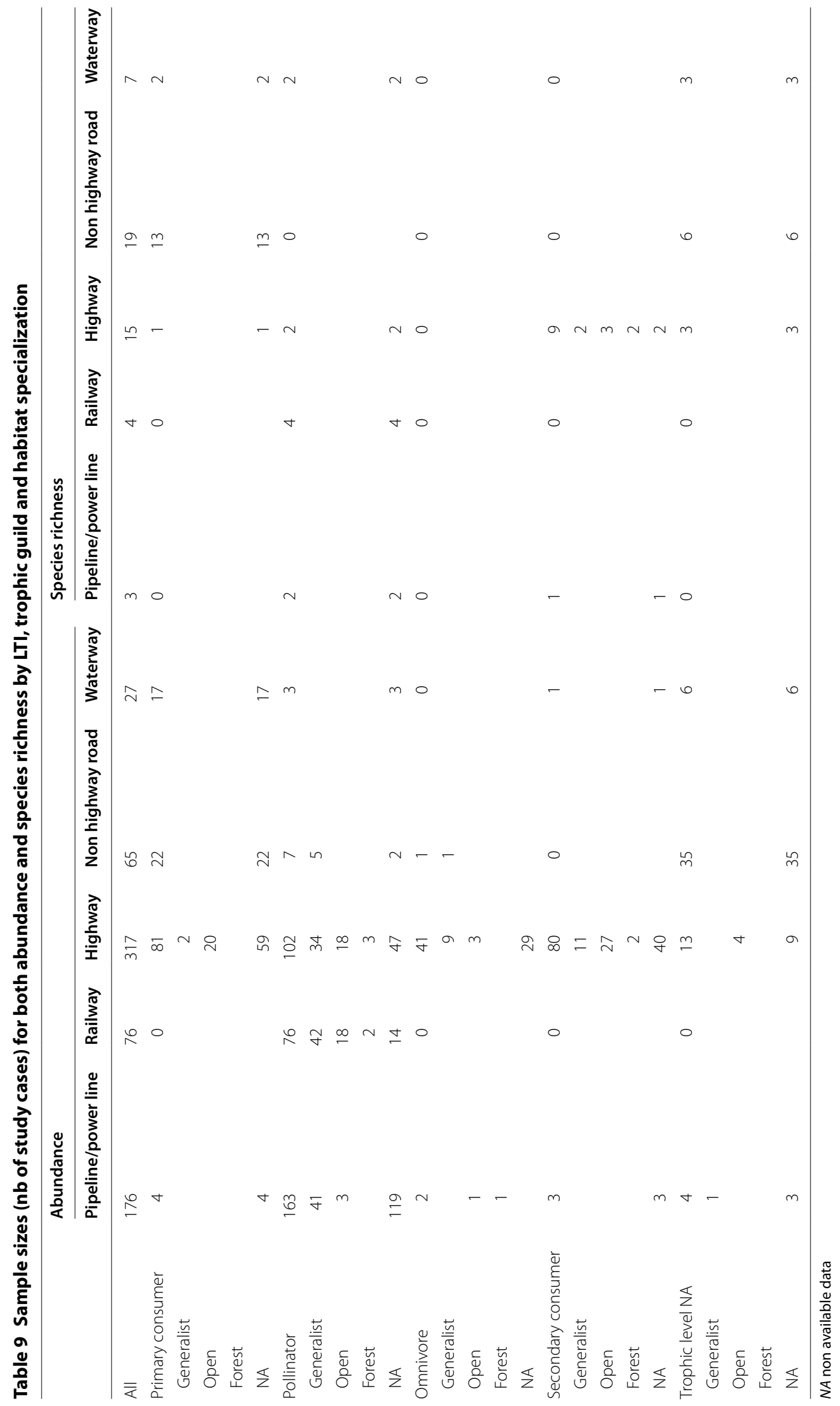


$\mathrm{p}<0.001, \mathrm{n}=661$ ), indicating variations in the differences of abundance between verges and habitats away from LTIs that could be explained by moderators. The funnel plot, the plot of the mean cumulative meta-analysis by publication year (Additional file 8) and the Rosenberg test fail safe number $\left(\mathrm{N}_{\mathrm{th}}=3315<\mathrm{N}_{\mathrm{obs}}=36,991\right)$ did not show obvious publication bias. The type of literature (grey/non-grey literature) had no effect on the effect sizes for abundance $\left(\mathrm{Q}_{\mathrm{M}}=4.181, \mathrm{p}=0.124, \mathrm{n}=661\right)$. None of the variables related to the design of the studies showed statistically significant effect (susceptibility to bias (low/medium): $\mathrm{Q}_{\mathrm{M}}=3.748, \mathrm{p}=0.154, \mathrm{n}=661$; study duration ( 1 year/more): $\mathrm{Q}_{\mathrm{M}}=4.069, \mathrm{p}=0.131$, $\mathrm{n}=661$; true spatial replication (yes $/ \mathrm{no}$ ): $\mathrm{Q}_{\mathrm{M}}=4.733$, $\mathrm{p}=0.094, \mathrm{n}=661$; sample location (random/not random): $\mathrm{Q}_{\mathrm{M}}=5.353, \mathrm{p}=0.069, \mathrm{n}=661$ ).

The overall grand mean weighted effect size for species richness was not statistically different from zero $(\mathrm{d}=0.269,95 \% \mathrm{CI}-0.049,0.587, \mathrm{p}=0.097)$. There was a statistically significant heterogeneity in the effect sizes $(\mathrm{Q}=243.75, \mathrm{p}<0.001, \mathrm{n}=48)$, indicating variation in the differences of species richness between verges and habitats away from LTIs. The funnel plot, the plot of the mean cumulative meta-analysis by publication year (Additional file 8) and Rosenberg test $\left(\mathrm{N}_{\text {th }}=250<\mathrm{N}_{\mathrm{obs}}=705\right)$ did not show obvious publication bias. The type of literature (grey/non-grey literature) had no effect on the effect sizes for species richness $\left(\mathrm{Q}_{M}=2.686, \mathrm{p}=0.261, \mathrm{n}=48\right)$. None of the variables related to the design of the studies showed statistically significant effect (susceptibility to bias (low/medium): $\mathrm{Q}_{\mathrm{M}}=2.498, \mathrm{p}=0.287, \mathrm{n}=48$; study duration ( 1 year/more): $\mathrm{Q}_{\mathrm{M}}=3.828, \mathrm{p}=0.148$, $\mathrm{n}=48$; true spatial replication (yes $/ \mathrm{no}$ ): $\mathrm{Q}_{\mathrm{M}}=4.162$, $\mathrm{p}=0.125, \mathrm{n}=48$; sample location (random/not random): $\left.\mathrm{Q}_{\mathrm{M}}=4.161, \mathrm{p}=0.125, \mathrm{n}=48\right)$.

\section{Effects of moderators on abundance}

We discarded the railway as a type of LTI from the analysis because the cases belonged to a unique study. For the same reason, some trophic levels were discarded (Figs. 7, 8).

There was no statistically significant interaction between habitat specialization and trophic level for the abundance of insects of the "highway" subset (model with interaction $\mathrm{AIC}=227.8$, model without interaction $\mathrm{AIC}=227.9$, Anova $\mathrm{p}=0.147$ ), nor between habitat specialization and LTI type for the abundance of pollinators (model with interaction AIC $=211.5$, model without interaction $\mathrm{AIC}=207.3$, Anova $\mathrm{p}=0.612$ ). We therefore pooled the cases to improve the statistical power in further analyses.

For highways, we found that the trophic level did not influence the response of insect abundance. Their abundance were similar in verges and in habitats away from LTIs (see statistical results in Fig. 7).

For non-highway roads, both pollinators and primary consumers were more abundant along verges than in habitats away from LTIs (Fig. 8), with a statistically significant stronger effect for pollinators (see statistical results in Fig. 7).

For waterways, the responses of primary consumers and pollinators were not statistically different $\left(\mathrm{Q}_{\mathrm{M}}=4.11, \mathrm{p}=0.13, \mathrm{n}=20\right)$. Pollinators seemed to be less abundant in waterway banks than in controls but this result was only based on 3 cases from 2 studies (Fig. 8).

For pipelines/power lines, we could only test pollinators and they had similar abundance between verges and controls.

Habitat contrast between verges and compared habitats did not influence our results: the interaction between habitat contrast and trophic level was not statistically significant for the abundance of insects in the "highway" subset (model with interaction AIC $=280.3$, model without interaction $\mathrm{AIC}=278.4$, Anova $\mathrm{p}=0.91$ ).

\section{Effects of moderators on species richness}

We detected a statistically significant effect of the LTI type on insect species richness: we found that insect species richness was greater on non-highway road verges than in controls away from non-highway roads (see statistical results in Fig. 7 and illustration in Fig. 9); whereas the species richness did not differ between verges and habitats away from waterways, pipelines/ power lines, highways and railways. However, we were not able to evaluate the response of each trophic level separately. Consequently, these results could be biased by an uneven distribution of trophic levels studied between LTI types. So, they should be taken with great caution and further research is needed to confirm our result.

The sensitivity analyses we performed show that our main results for abundance and species richness were robust to the fact that some case studies may not be fully independent as they came from the same primary study (Additional file 9).

\section{Discussion \\ Evidence of effects}

Overall, we found variations in the results of the studies that deal with our six specific questions, and outcomes seemed difficult to predict. However, we did find some patterns, as shown by the Table 10 that summarizes the main results coming from the narrative syntheses.

We identified major knowledge gaps for the three specific questions related to insect dispersal in verges (questions 3, 4 and 6 in Table 10). Technical constraints that 


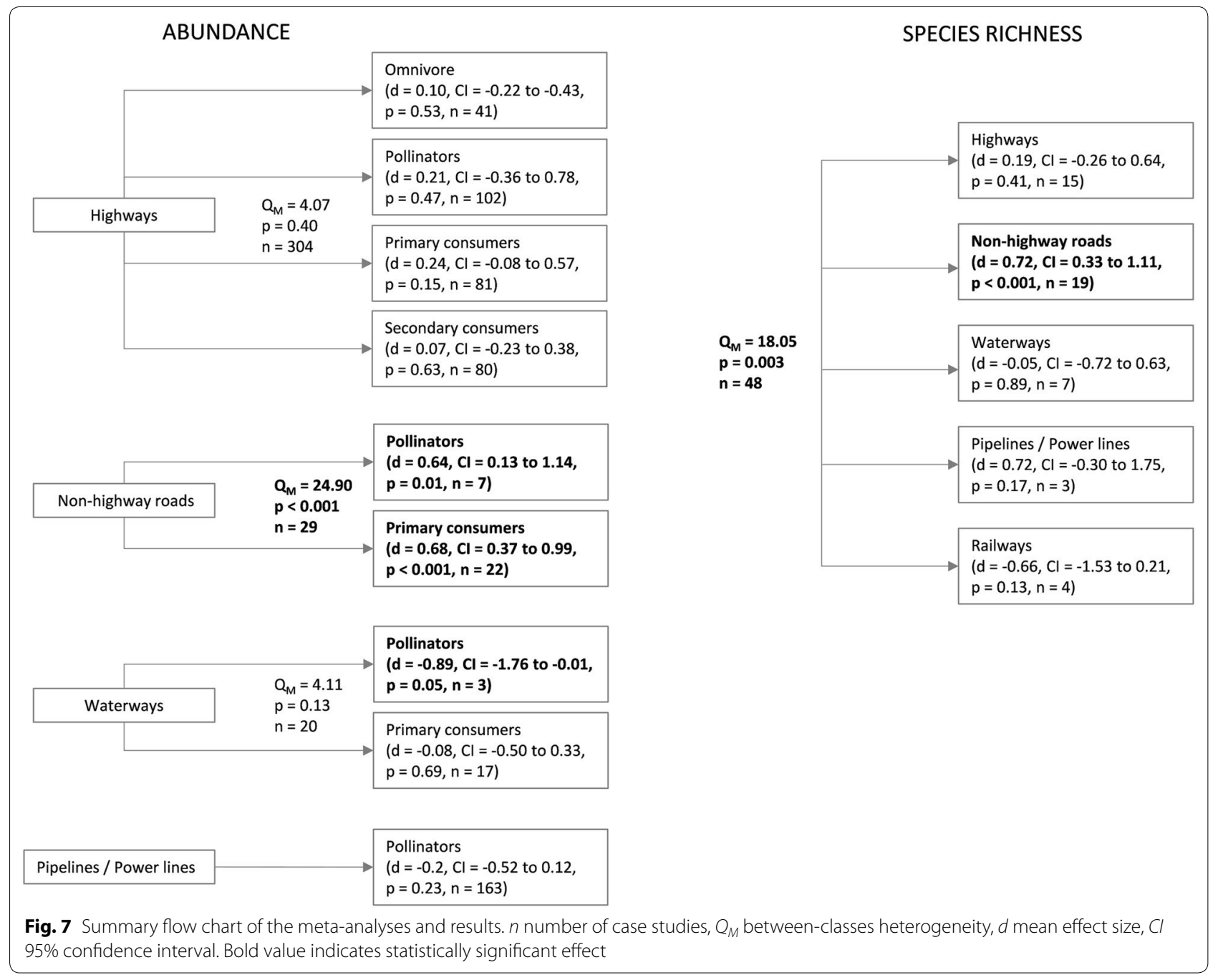

challenge the studies of insect movements are not the only factor responsible for these gaps. Indeed, if the small body size of the insects has so far precluded the use of most radio-tracking equipment, it is still possible to evaluate their dispersal through mark-recapture method, genetic analysis or direct observation. There is a number of studies that evaluated insect movements near LTIs [124], but nearly all of them aimed at evaluating the barrier effect of LTIs, hence focusing on transversal movements but not on longitudinal ones [125-127]. Therefore, the fragmentation effect of LTIs seems to have received all scientific and monitoring interests, leaving their potential corridor effects largely unexplored, at least for insects in temperate regions.

The negative to neutral effect of the artificialization of the LTI (use of non-natural material, channelization) is not surprising and converges to the general trend of negative effects of habitat loss, habitat degradation and ecosystem functioning disruption on biodiversity [128]. The positive to neutral effect we found for management practices that aim to restore a more "natural" vegetation along verges is of conservation interest for LTI owners. The positive effect is driven by studies where management practices consisted in getting rid of exotic invasive plants species along waterways. We were not able to draw a general trend for the effect of grazing and mowing of the LTI verges on insect biodiversity. More studies are needed to conclude on these vegetation management practices and advise those that favor insect biodiversity.

Previous reviews focused on the comparison of animal abundance near LTIs and in other habitats. Indeed, Muñoz et al. [19] found that in 50\% of the studies that they reviewed, the abundance and/or diversity of insects increased from the roadsides to the non-altered habitats, implying a negative effect of the roads. Our results were more nuanced and even contrasted (question \#2 in Table 10). This discrepancy can be explained by the fact that we included not only road verges, and that, contrary 


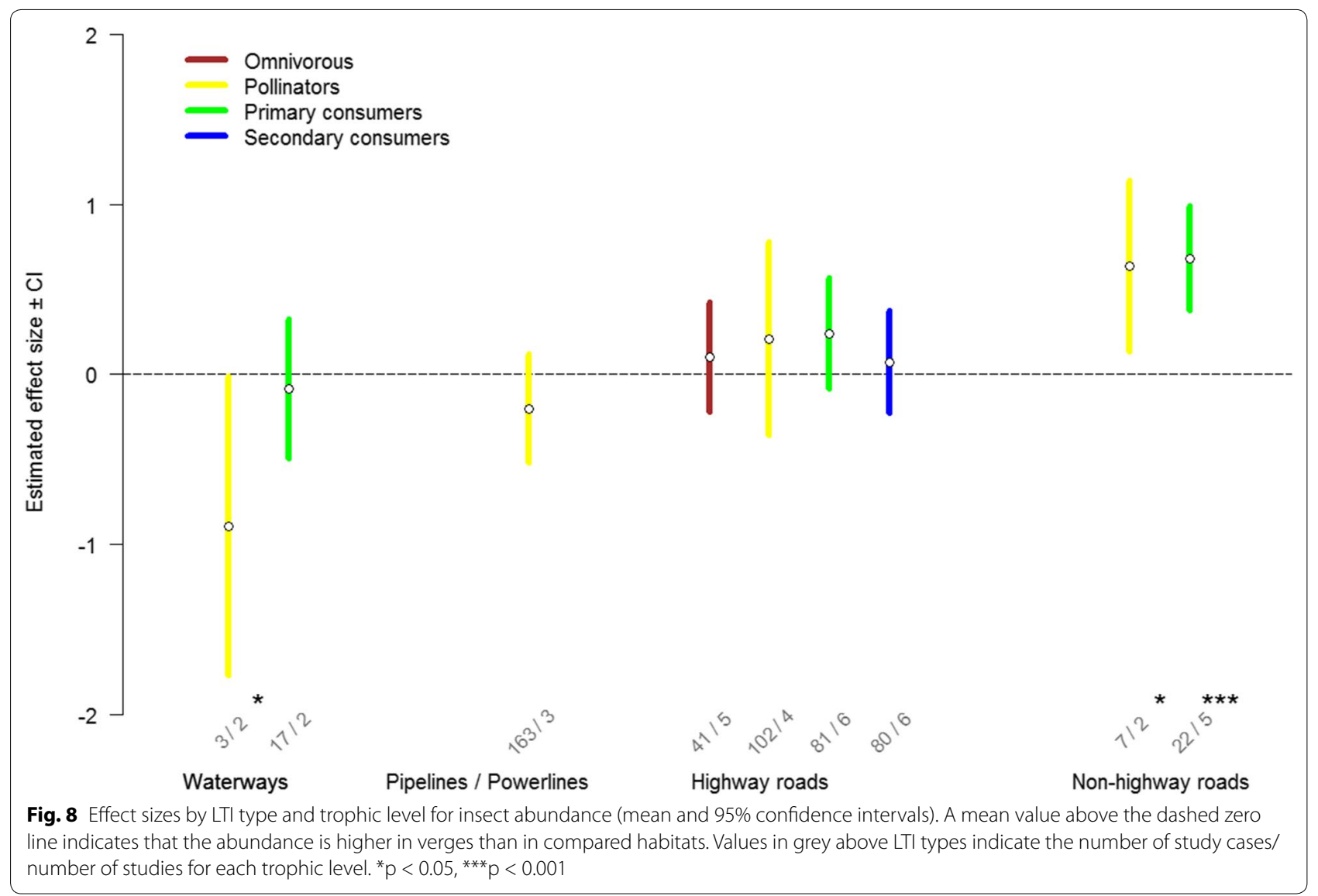

to Muñoz et al. [19], we did not restrict our synthesis to studies that compare insect abundance and diversity at increasing distances from the LTIs.

Fahrig and Rytwinski's review [38] found that animals had a positive or neutral response to roads when (i) species are attracted to roads for an important resource (e.g. food) and are able to avoid oncoming cars, and (ii) species do not avoid traffic disturbance but do avoid roads, and whose main predators show negative populationlevel responses to roads.

Our meta-analyses suggest that highway roadsides are not similar to non-highway roadsides in terms of insect abundance and species richness. Indeed, pollinators and primary consumers are more abundant in non-highway roadsides than in habitats away from LTIs whereas their abundances do not differ between highway roadsides and controls. The difference between small and large roads might be explained by the positive correlation between traffic volume and insect collisions [19], and a higher edge effect on highways that lowers the carrying capacity of their verges. However, long-term studies of population dynamics are missing to disentangle potential ecological traps from actual positive effects of non-highway road verges on insect abundance [129].

Our narrative synthesis showed that the nature of the landscape surrounding LTIs can influence the biodiversity hosted by the verges. The negative effect of land uses related to human pressure (i.e. urbanization and agriculture) and the positive effect of forests and (protected) natural habitats in the surroundings concur with the "habitat amount hypothesis" supported by Fahrig [130]. Indeed, urbanization and agriculture development around LTI verges reduce the amount of suitable habitats at the landscape scale and, consequently, the insect species pool able to colonize the verges. The opposite process is true for undisturbed (or less disturbed) natural habitats that increase habitat availability and the presence of source populations.

\section{Reasons for heterogeneity}

One of the main reasons for heterogeneity is the variety of comparators used among studies within each of our six specific questions. Indeed, the studies that compared insect biodiversity between LTI verges and 


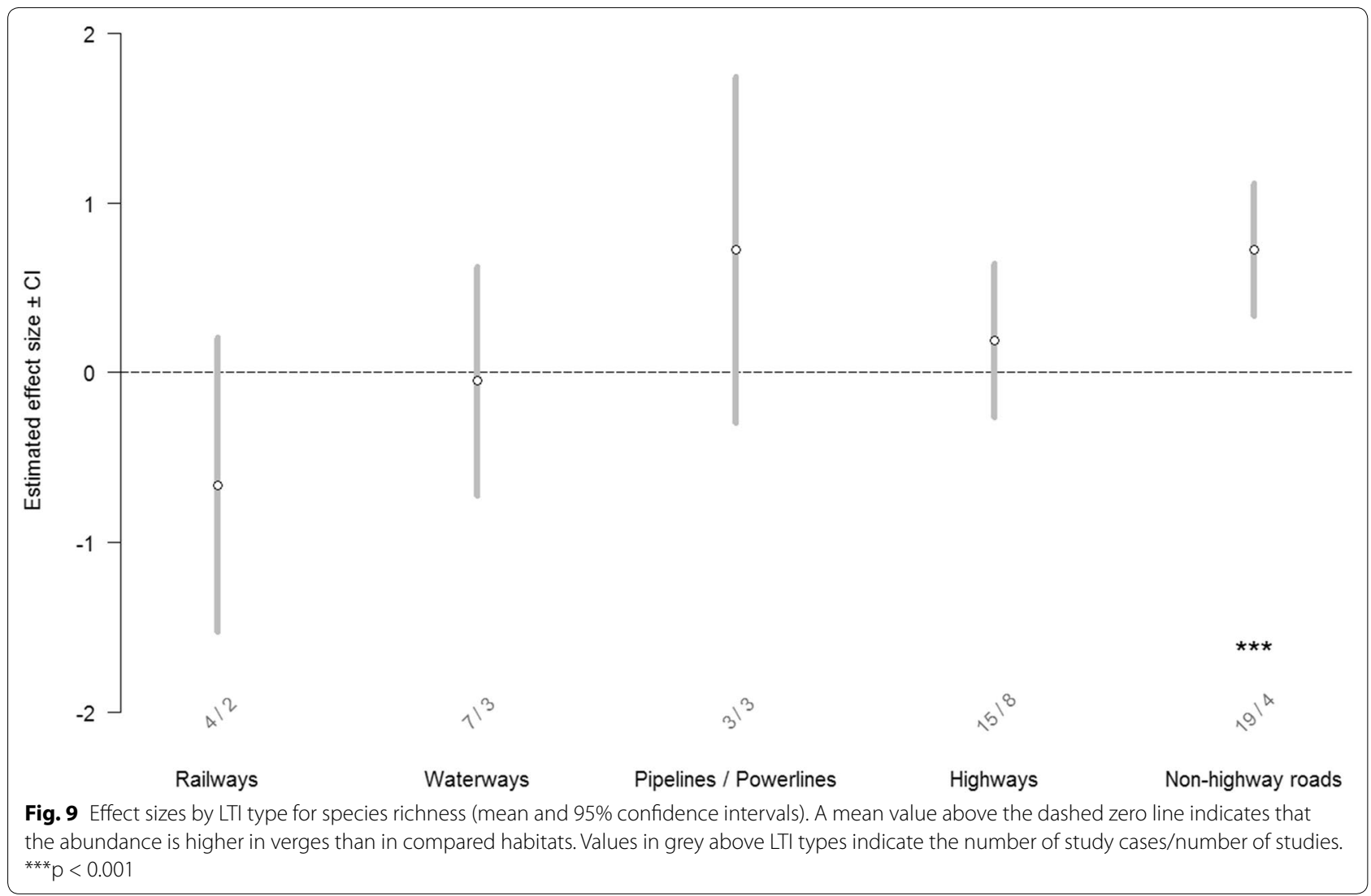

habitats in proximity differ regarding the amount of contrast between the two habitats; for example in terms of resources used by the insects. Similarly, the type of management practices also greatly influences the carrying capacity of LTI verges. For instance, the effect of invasive plant removal along roadsides would greatly differ from the effect of mowing. Thus, we grouped studies that focused on the same kind of management. Nevertheless, there was still a large amount of heterogeneity between the interventions (e.g. various mowing regimes).

Similarly, there was a wide range of designs, landscape metrics and spatial scales used in studies that evaluated the influence of the surrounding landscape on the biodiversity hosted by the LTI verges.

In addition, the type of LTI studied and the type of LTI verge are important sources of variation. For instance, waterways and power lines present major differences in terms of transportation, physical properties, shapes, vegetation type of their verges and hence, species hosted. The LTI verges also differ in their management, use, history, width, etc. Therefore, their quality and carrying capacity for insect populations are not homogeneous even among LTI verges of a given type.
Another non-negligible cause of disparity between the results of the studies is the variety of species in terms of biology, ecology, physiology and functional traits. Indeed, insects encompass a wide range of taxa, from aquatic caddisflies, major pollinators, to agricultural pests such as some aphids. Each species or taxonomic group has its own requirements, and as a result, a management practice favoring species with certain traits could be detrimental for other species.

In the meta-analyses, the variation in effect sizes observed most likely reflects the differences between LTI types and the ecological requirements of the taxa. Unfortunately, the small number of studies together with the scarcity in the description of the LTIs, LTI verges and studied habitats, did not allow us to conduct quantitative examination of the influence of environmental conditions at both verges and compared habitats, or variables related to LTI characteristics. Thus, we were not able to go deeper in the prospection of the reasons for heterogeneity.

Lastly, as metrics of biodiversity, species richness and abundance suffer from a kind of blindness as they consider all the species to be equal. To overcome this 


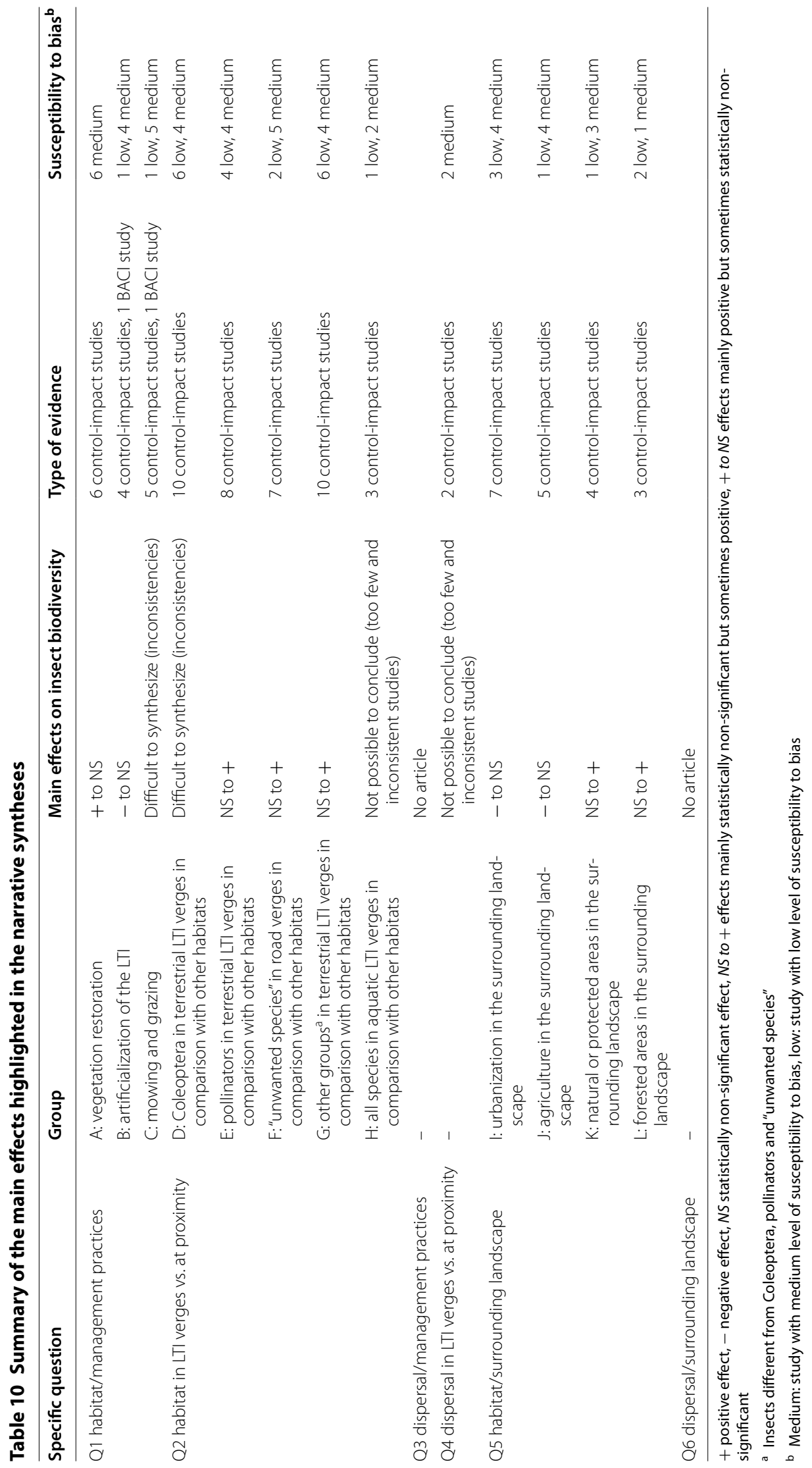


limitation, we tested the effect of trophic level and habitat specialization. Unfortunately, we were not able to analyze the effect of species conservation status, and a quantitative evaluation of the difference in species composition was beyond the objective of this review.

\section{Review limitations}

Most of the studies included in the map are based on data collected within 1 or 2 years, with very few long-term studies. For insects, which can be subject to extreme inter-annual population variations, this deficiency can limit the conclusions of individual studies. In addition, there are few highly replicated large scale experiments, providing increased robustness and reliability of the results. Some studies focused on very restricted areas and did not include a true spatial replication of LTI verges. Therefore, their conclusions are context-dependent and difficult to extrapolate to other areas. Moreover, only some of the studies included in this review incorporate data collection before the intervention. This can be problematic, especially to evaluate the influence of management practices, because it is not possible to distinguish real management effect from pre-existing differences between control and managed sites. Moreover, besides the limitations of the individual studies included in this review, we note a lack of common research protocol for each specific question. Indeed, researchers measured several aspects of insect biodiversity in dissimilar ways, which challenges the comparison of results among studies.

Our systematic review focuses on the potential of linear transportation infrastructure verges for insects, therefore, for aquatic linear structures our objective was to only select articles on navigable waterways. Nevertheless, navigability of the waterways is rarely provided by authors and there is not international database that references this information. Thus, we applied inclusion/ exclusion criteria (Table 6) that sound relevant to restrict included articles to major waterways, implicitly assuming that they might be navigable. We are aware that this solution is not perfect: some articles about navigable waterways could have been excluded and others about non-navigable waterways could have been included. In addition, it is quite tricky to distinguish study sites within waterway verges from those out of the verges. Contrary to the other LTIs, the concept of verge for a waterway can be ambiguous because of the fluctuation of the water level throughout seasons. As a consequence, we recommend authors to add these indications in their study site description.
We limited our narrative syntheses to the comparisons that were statistically tested. This restriction led us to put aside some grey literature documents without statistics, as well as some results from old scientific articles for which we were not sure that the comparisons provided were statistically tested. Likewise, papers comparing more than two categories, which do not provide results for each comparison (Kruskal-Wallis test, Anova or linear regression without post hoc tests), challenged our ability to include them. In several studies, particularly older ones, statistically non-significant results are often not shown, possibly inducing publication bias. However, in the meta-analyses, fail-safe numbers indicated that our results were sufficiently robust. Another issue stems from the studies that evaluate the effects of multiple landscape metrics simultaneously, using model selection in additive regression (this is the case for two of the studies [31, 120] included in the narrative synthesis for the question \#5). Indeed, because of the collinearity between metrics, a landscape variable that is not retained in the best model does not necessarily have no effect. To properly test the effect of each landscape metric, it would have been necessary to compare the null model with the corresponding univariate model.

For 5 of our 6 specific questions, the small number of articles and the diversity of the studies we captured limited our ability to implement meta-analyses. For that reason, we reviewed the evidence using narrative syntheses. However, narrative syntheses cannot provide reliable information on the magnitude or consistency (homogeneity) of the results of the studies. In addition, when sample size and magnitude are low and variability is high, the studies are unable to detect a real response, thus narrative syntheses are more prone to negative bias because the ability to detect real effects is limited [68]. Consequently, the conclusions we provided for these questions should be taken with caution, and we recommend more research on these topics to allow meta-analyses in the future.

Our meta-analysis for species richness was based on a limited number of study cases in comparison to the meta-analysis for abundance $(\mathrm{N}=48$ and $\mathrm{N}=661$ respectively). At the same time, the grand mean effect size was twice as high for the species richness as for the abundance $(\mathrm{d}=0.269$ and $\mathrm{d}=0.174$ respectively). Consistent with our findings that publication bias is unlikely to affect our conclusions, differences in effectsizes between richness and abundance is likely ecologically relevant and we hypothesize that a higher number of primary studies reporting data on species richness may allow us to detect a statistically positive grand mean 
effect size for species richness as primary studies will accumulate.

For abundance, some of the meta-analyses that we conducted were based on a limited number of studies, in particular when evaluating the effect of trophic level. Together with the uneven distribution of the effect sizes between moderator categories, it precluded the investigations of the influence of habitat specialization and of the contrast between verges and controls in a proper hierarchical approach. Moreover, we were unable to further investigate the influence of the contrast between LTI verges and habitats away from LTIs in terms of food and nesting resources or abiotic factors, because there was not enough information reported within studies to do so.

To save time during screening, we decided to exclude articles that did not provide any abstract. This is not consistent with CEE guidelines which recommend including them in the full text assessment. We acknowledge that this can be a potential source of bias. However, a first examination of a sample of these documents showed that the publication type was unsuitable for all of them (editorial, letter, review, policy recommendation, meeting report...), so that they would have been eventually excluded in subsequent steps.

Although it is good practice, we were unable to test the consistency of reviewers' inclusion/exclusion decisions at the full text stage due to logistical constraints and time limitation. Thus, despite clarifications and adjustments between reviewers before the full text assessment, an observer effect at the full text stage might be a potential source of bias in our review.

The language restriction we applied (French and English), biased the studies towards English and French speaking countries. However, we do not believe that the geographic bias created by the language restriction would bias the outcomes of our review as linear transportation infrastructure verges are essentially the same across the temperate zone.

Roads encompass 40 out of the 63 studies included in the narrative syntheses and 416 out of the 709 effect sizes extracted for the meta-analyses. The under-representation of the LTIs other than roads limits the relevance of our systematic review for these LTIs. Moreover, the taxonomic bias restricts the conclusions we can draw for taxa other than Coleoptera, Hymenoptera and Rhopalocera. Finally, because it focused on insects, we would like to emphasize that this review only represents a partial estimate of the potential of LTI verges as habitat and corridor for biodiversity. Therefore, we highlight the importance of broadening the review of evidence to other taxonomic groups such as plants and vertebrates, and to climatic regions different from the temperate area.

\section{Conclusion \\ Implication for policy/management}

Overall, the evidence is too weak to inform policy or management with specific detailed recommendations on the concrete questions LTI managers have about their infrastructures. For example, evidence was too small and scattered to answer the following questions: "Can LTI verges serve as movement corridor for pollinators?" or "What type of river embankment favors insect biodiversity?".

However, some key results can inform policy makers and LTI managers. First, LTI verges are not "deserts". They can harbor similar to higher insect biodiversity than habitats away from LTIs. Thus, this result gives another perspective for LTI managers: LTIs are not always detrimental for biodiversity, in certain conditions they can contribute to biodiversity conservation. Given the large amount of LTI verges, especially in Western Europe, policy makers should consider these landscape elements in biodiversity conservation politics. This advice concurs with the "land sharing" paradigm, which considers the potential of all land uses in biodiversity conservation and reconciles nature and human activities [131]. It complements "land sparing" politics where biodiversity conservation is restricted to small protected areas [131].

Secondly, management practices which preserve or restore LTI "naturalness" seem to benefit insect abundance and diversity in verges. Favoring the use of natural material in the verges, preserving the natural functioning of the ecosystem, and promoting/preserving the development of native vegetation instead of exotic or mono-specific vegetation along verges are sensible general pieces of advice.

Finally, we showed that insect biodiversity hosted by LTI verges depends on the landscape context. In consequence, LTI managers should take this effect into account when choosing where to dedicate special efforts for biodiversity conservation.

\section{Implication for research and monitoring}

In regards to the research gaps we highlighted, we advise to dedicate resources on the monitoring of insect movements along LTI verges. These studies are crucial to evaluate if insects can benefit from LTI verges as corridors, and if management practices or landscape context are likely to affect the corridor effectiveness. In addition, it is necessary to devote more efforts on LTIs 
other than roads, especially on railways, power lines and pipelines (proportionally to their cumulative length). Moreover, scientists should focus on other insect groups than Coleoptera, Lepidoptera and Hymenoptera [19], which only represent a small part of the huge insect biodiversity.

Another interesting avenue for future research is the contribution of LTI verges to biodiversity at the landscape scale. Indeed, if LTI verges might support less abundant and diverse insect communities in human modified landscapes (urban or agricultural), they can constitute the last relict of degraded semi-natural habitats and refuges for wildlife. To investigate this aspect, it will be necessary to compare the contribution of LTI verges in the total landscape biodiversity (gamma diversity) along a land-use intensity gradient.

Finally, we provide some suggestions to improve study standardization and data reporting:

- clearly define the LTI verges (vegetation type, management, resources, adjacent habitat, landscape context, history) and clearly define the habitats compared to the LTI verges (Questions \# 2 and 4) and if possible evaluate the contrast between LTI verges and habitats away from verges in terms of vegetation type and quality (resource availability).

- be very clear in "Methods" section on how the experiment was done (study duration, time since construction of the LTI, type of replication, sampling location: distance from the LTI and the adjacent habitat ...).

- include more information in table and figure captions (such as the number of replicates that the means are based on, and the type of variation that is shown).

- present all results, even statistically non-significant ones (in appendix if necessary).

- make raw data available (appendix or deposition in public data repositories).

- include information about species requirement and biology (habitat specialization, trophic level, dispersal capacity, conservation status, etc.)

- increase the study duration, the number of true spatial replicates and the spatial extent of the studies.

- favor data collection before the intervention when studying the effect of management practices (BACI, BAI designs).

- if possible, adopt a gradient approach to investigate variations in response to management intensity (e.g. mowing frequency).

\section{Additional files}

Additional file 1. List of search terms.

Additional file 2. Studies of the test list identified by subject experts with indications of indexation in Web of Science Core Collection and Zoological Records and collection with the search strings.

Additional file 3. Search strings used in Google Scholar.

Additional file 4. Database of the studies: identifier (ID_doc), full reference, publication type, language, specific question addressed, country, region, GPS coordinates, biological group, LTI, LTI verge, comparison, study design, outcomes, susceptibility to bias and indication of the articles included in narrative syntheses and meta-analyses.

Additional file 5. List of articles with missing full text and articles excluded at full-text screening and critical appraisal with reasons.

Additional file 6. Tables synthesizing key results of the studies included in the narrative syntheses: full reference, country, insect group, LTI, LTI verge, comparison, key results and groups used to synthesize the evidence in the narrative syntheses.

Additional file 7. Data included in the meta-analyses: study case identifier (Case.ID), study identifier (ID_doc), short reference, susceptibility to bias, randomisation, true spatial replication, type of literature, study duration, country, ILT, habitat contrast, insect order, insect groups, trophic level, habitat specialization, conservation status, response variable, data extracted for treatment ( $\mathrm{t}$ ) and control (c) from studies ( $\mathrm{N}$ : sample sizes, MEAN: mean, VAR: measure of variability, BOXPLOT first/third quartile: first and third quartiles), Stat. test: identity, direction, effect and pvalue for treatment/control comparison given within studies, Ncorr: total sample size corrected for non-independent cases, effect size (d) and variance (var).

Additional file 8. Results of the publication bias analysis for abundance and species richness: funnel plots and plots of the mean cumulative meta-analysis by publication year.

Additional file 9. Sensitivity analysis of the effect of multiple case studies per primary study.

\section{Abbreviations}

LTI: linear transportation infrastructure; CILB: Club des Infrastructures Linéaires \& Biodiversité; MEEM: Ministère de l'Environnement de l'Énergie et de la Mer; ITTECOP: Infrastructures de Transport Terrestre, Écosystèmes et Paysage; FRB: Fondation pour la Recherche sur la Biodiversité; MNHN: Muséum national d'Histoire naturelle; Irstea: Institut national de recherche en sciences et technologies pour l'environnement et l'agriculture; Cerema: Centre d'études et d'expertise sur les risques, l'environnement, la mobilité et l'aménagement; BA: before/after; Cl: comparator/intervention; BACl: before/after/comparator/intervention; AJ: Arzhvaël JEUSSET; AC: Aurélie COULON; AV: Anne VILLEMEY; BC: Bastien CASTAGNEYROL; EG: Eric GUINARD; EJ: Emmanuel JASLIER; ELM: Eric LE MITOUARD; FFL: Frédérique FLAMERIE DE LACHAPELLE; HJ: Hervé JACTEL; IW: Isabelle WITTE; JT: Julien TOUROULT; ND: Nadine DENIAUD; MV: Marianne VARGAC; RS: Romain SORDELLO; SV: Sylvie VANPEENE; VRo: Véronique ROY; VRa: Vanessa RAUEL; YB: Yves BERTHEAU.

\section{Authors' contributions}

$\mathrm{RS}, \mathrm{YB}, \mathrm{AC}$ and JT conceived the review question. AJ, MV, RS and SV undertook the experts request for scientific key articles. AJ, RS, MV, AC, YB and SV validated the test list. MV, FFL, AJ, RS, VRo, EJ, ND, AC, YB and SV contributed to the database selection. AJ, MV, RS, AC, YB, SV and FFL contributed to the search terms brainstorming. AJ, MV, RS, FFL, VRo, EJ and ND undertook the search comprehensiveness assessment and equation selection. RS and AJ organized the external experts workshop for critical appraisal and AC, YB, SV 
attended it. Literature stemming from Web of Science and Zoological Records was screened on title, summary and full text by AJ, RS, MV, SV, AC, YB, EG, ELM, VRa and AV. Literature stemming from the call to subject experts was screened on title by MV and on full-text by EG, ELM and VRa. Literature stemming from Google Scholar was screened on title and full-text by EG, ELM and VRa. Critical appraisal and extraction of the results in qualitative tables were made by $A V$, $A J, R S, M V, S V, A C$ and YB. AV performed data extraction for the meta-analyses. IW and $A V$ implemented the meta-analyses and $B C$ and $\mathrm{HJ}$ offered their support on specific questions. RS was the scientific coordinator of the project. FFL, VRo, EJ, ND offered support for the bibliographical stage of the study. All authors joined regular steering committee meetings. AV and AJ equally contributed to the writing of the manuscript. Romain Sordello scientific coordinator of the project. All authors read and approved the final manuscript.

\section{Author details}

${ }^{1}$ UMS 2006 PatriNat, Centre National de la Recherche Scientifique (CNRS), Muséum national d'Histoire naturelle (MNHN), Agence Française pour la Biodiversité (AFB), 75005 Paris, France. ${ }^{2}$ Centre d'Ecologie et de Sciences de la COnservation (CESCO), Muséum national d'Histoire naturelle (MNHN), 75005 Paris, France. ${ }^{3}$ Institut national de la recherche agronomique (Inra), SPE, route de Saint Cyr, 78026 Versailles cedex, France. ${ }^{4}$ PSL Research University, CEFE UMR 5175, CNRS, Université de Montpellier, Université Paul-Valéry Montpellier, EPHE, Laboratoire Biogéographie et Ecologie des vertébrés, 1919 route de Mende, 34293 Montpellier, France. ${ }^{5}$ Institut national de recherche en sciences et technologies pour l'environnement et l'agriculture (Irstea), 13182 Aix-en-Provence, France. ${ }^{6}$ Laboratory of Forest Entomology \& Biodiversity, UMR 1202 BIOGECO, INRA, 33610 Cestas, France. ${ }^{7}$ BIOGECO, UMR 1202, University of Bordeaux, 33400 Talence, France. ${ }^{8}$ Muséum national d'Histoire naturelle (MNHN), 75005 Paris, France. ${ }^{9}$ Université Paris 6 Pierre et Marie Curie (UPMC), 75005 Paris, France. ${ }^{10}$ Centre d'études et d'Expertises sur les Risques, I'Environnement, la Mobilité et l'Aménagement (Cerema), DTer Sud Ouest DAIT GBMN, CS60013, 33166 Saint-Médard-en-Jalles, France.

\section{Acknowledgements}

The authors thank the CILB and the MEEM for funding this research project, the ITTECOP research incentive program for their logistical help and the FRB for their methodological support on systematic reviews and the organization of the steering committee meetings.

The authors thank Barbara Livoreil (FRB) who contributed to the validation of the test list, the database selection, the search terms brainstorming and the external experts' workshop for critical appraisal. The authors also thank Patrick Pacevicius (Cerema) for his contribution to the screening and critical appraisal of articles from the call to subject experts and Google Scholar. The authors thank Jean-François Godeau for his contribution to critical appraisal of articles.

The authors also thank Leyli Borner and Isabelle Le Viol (MNHN), Jean Carsignol (Cerema), Violette Le Féon (Institut national de la recherche agronomique), Jean-François Godeau (Life Elia project), Sébastien Filoche (Conservatoire botanique national du Bassin parisien) and Damien Picard (Université d'Angers) for their contribution to the critical appraisal workshop.

Finally, the authors thank the "Union internationale pour la conservation de la nature France", the MEEM, the "Fédération des Parcs Naturels Régionaux", the "Centre d'études et d'expertise sur les risques, l'environnement, la mobilité et l'aménagement", the "Société Anonyme Française d'Étude de Gestion et d'Entreprises", the "Société d'études techniques et économiques", the ITTECOP, the CILB and all the contacted experts for sending them scientific and grey literature and putting them in contact with other experts.

\section{Competing interests}

The authors declare that they have no competing interests.

\section{Availability of data and materials}

The datasets supporting the conclusions of this article are included within the article and its additional files.

\section{Consent for publication}

Not applicable.

\section{Ethics approval and consent to participate}

Not applicable.

\section{Funding and support}

The project was supported by three organizations: the CILB, the MEEM and the FRB. It was funded by the CILB, which is composed of the following LTI managing organizations: Réseau Ferré de France (now named SNCF Réseau), Voies Navigables de France, Réseau de Transport d'Électricité, GRT Gaz, Transport et Infrastructures Gaz France and Électricité Réseau Distribution France (now ENEDIS), EIFFAGE and Association des Sociétés Françaises d'Autoroutes. Besides, the MEEM and ADEME (Agence de l'environnement et de la maîtrise de l'énergie) supported the project through the ITTECOP program which constituted a framework for the project, offered a partial financial contribution to the project and provided funding through the Cerema participation. Finally, the FRB gave the research team methodological support for the realization of the systematic review.

\section{Candidate selection}

The MNHN was selected by the CILB, the ITTECOP and the FRB to conduct the systematic review following a public call for projects, for which the MNHN had submitted a proposal.

\section{Project course}

The ITTECOP was met once by RS and AJ to present the first stages of the project and ask for directories of experts of transportation ecology. LTI managing organizations, the ITTECOP and the FRB were met twice by RS, AJ, MV, JT, $A C, Y B, S V$ during the course of the project to expose them the progress of the project. LTI managing organizations funding the project were also met once before the redaction of the present manuscript, by $\mathrm{RS}, \mathrm{AJ}$ and $\mathrm{MV}$, in order to clarify their needs for information and the inherent scientific question.

The FRB, through its representative Barbara Livoreil, had a particular role in the first stages of the project as it is a backer of the project, a member of the research team and a representative of the Collaboration for Environmental Evidence, which approves environmental systematic reviews. Nevertheless, the role of the FRB in the decisions made by the research team remained advisory and the present document was proofread by independent peer-reviewers.

All project decisions regarding the design, the collection, the analysis and the interpretation of data were made freely by the authors of the study. All statements made in this report are under the responsibility of the authors and do not necessarily represent the views of the CILB or of the ITTECOP.

The review manuscript was sent to the funding body for information when submitting it for publication.

\section{Other projects}

After responding to a call for tender, the MNHN was selected to conduct another project than the present one, named Trans-fer, which was also funded by Réseau Ferré de France (a member of the CILB) and the MEEM. Trans-fer ended before the beginning of the present project and the two projects were steered independently.

\section{Publisher's Note}

Springer Nature remains neutral with regard to jurisdictional claims in published maps and institutional affiliations.

Received: 27 April 2017 Accepted: 5 January 2018

Published online: 05 February 2018

\section{References}

1. Butchart SHM, Walpole M, Collen B, van Strien A, Scharlemann JPW, Almond REA, et al. Global biodiversity: indicators of recent declines. Science. 2010;328:1164-8.

2. Dirzo R, Raven PH. Global state of biodiversity and loss. Annu Rev Environ Resour. 2003;28:137-67.

3. Franklin AB, Noon BR, George TL. What is habitat fragmentation? Stud Avian Biol. 2002;25:20-9.

4. European Environment Agency. Landscape fragmentation in Europe: joint EEA-FOEN report. Copenhagen: European Environment Agency; 2011. 
5. MacArthur RH, Wilson EO. The theory of island biogeography. REVRevised. Princeton: Princeton University Press; 1967. http://www.jstor. org/stable/j.ctt19cc1t2. Accessed 23 June 2017.

6. Krauss J, Bommarco R, Guardiola M, Heikkinen RK, Helm A, Kuussaari $M$, et al. Habitat fragmentation causes immediate and time-delayed biodiversity loss at different trophic levels. Ecol Lett. 2010;13:597-605.

7. Fahrig L. Effects of habitat fragmentation on biodiversity. Annu Rev Ecol Evol Syst. 2003;34:487-515.

8. Berg A. Diversity and abundance of birds in relation to forest fragmentation, habitat quality and heterogeneity. Bird Study. 1997;44:355-66.

9. Davies KF, Margules CR. Effects of habitat fragmentation on carabid beetles: experimental evidence. J Anim Ecol. 1998;67:460-71.

10. van den Berg LJL, Bullock JM, Clarke RT, Langston RHW, Rose RJ. Territory selection by the Dartford warbler (Sylvia undata) in Dorset, England: the role of vegetation type, habitat fragmentation and population size. Biol Conserv. 2001;101:217-28.

11. Cushman SA. Effects of habitat loss and fragmentation on amphibians: a review and prospectus. Biol Conserv. 2006;128:231-40.

12. Zhou Y-B, Yang M-X, Yihuo W, Liu G, Wang H, Wei J-G, et al. Effect of habitat fragmentation on the schistosome-transmitting snail Oncomelania hupensis in a mountainous area of China. Trans R Soc Trop Med Hyg. 2011:105:189-96.

13. Fischer J, Lindenmayer DB. Landscape modification and habitat fragmentation: a synthesis. Glob Ecol Biogeogr. 2007;16:265-80.

14. Kuussaari M, Bommarco R, Heikkinen RK, Helm A, Krauss J, Lindborg R, et al. Extinction debt: a challenge for biodiversity conservation. Trends Ecol Evol. 2009;24:564-71.

15. Laurance WF, Lovejoy TE, Vasconcelos HL, Bruna EM, Didham RK, Stouffer PC, et al. Ecosystem decay of Amazonian forest fragments: a 22-year investigation. Conserv Biol. 2002;16:605-18.

16. Antrop M. Landscape change and the urbanization process in Europe. Dev Eur Landsc. 2004;67:9-26.

17. Laurance WF, Clements GR, Sloan S, O'Connell CS, Mueller ND, Goosem M, et al. A global strategy for road building. Nature. 2014;513:229-32.

18. Karlson M, Mörtberg U. A spatial ecological assessment of fragmentation and disturbance effects of the Swedish road network. Landsc Urban Plan. 2015:134:53-65.

19. Muñoz PT, Torres FP, Megías AG. Effects of roads on insects: a review. Biodivers Conserv. 2015;24:659-82.

20. Vos CC, Chardon JP. Effects of habitat fragmentation and road density on the distribution pattern of the moor frog Rana arvalis. J Appl Ecol. 1998:35:44-56.

21. Bujoczek M, Ciach M, Yosef R. Road-kills affect avian population quality. Biol Conserv. 2011;144:1036-9.

22. Glista DJ, DeVault TL, DeWoody JA. Vertebrate road mortality predominantly impacts amphibians. Herpetol Conserv Biol. 2008;3:77-87.

23. Rautenstrauch KR, Krausman PR. Preventing mule deer drownings in the Mohawk canal, Arizona. Wildl Soc Bull. 1989;17:280-6.

24. Gerlach $G$, Musolf K. Fragmentation of landscape as a cause for genetic subdivision in bank voles. Conserv Biol. 2000;14:1066-74.

25. Zachos FE, Althoff C, Steynitz YV, Eckert I, Hartl GB. Genetic analysis of an isolated red deer (Cervus elaphus) population showing signs of inbreeding depression. Eur J Wildl Res. 2007;53:61-7.

26. Keller I, Nentwig W, Largiader CR. Recent habitat fragmentation due to roads can lead to significant genetic differentiation in an abundant flightless ground beetle. Mol Ecol. 2004;13:2983-94.

27. Beier P, Noss RF. Do habitat corridors provide connectivity? Conserv Biol. 1998;12:1241-52.

28. Gilbert-Norton L, Wilson R, Stevens JR, Beard KH. A meta-analytic review of corridor effectiveness. Conserv Biol. 2010;24:660-8.

29. Bennett AF. Linkages in the landscape: the role of corridors and connectivity in wildlife conservation. Cambridge: IUCN Publ; 2003.

30. Heller NE, Zavaleta ES. Biodiversity management in the face of climate change: a review of 22 years of recommendations. Biol Conserv. 2009;142:14-32

31. Moroń D, Skórka P, Lenda M, Rożej-Pabijan E, Wantuch M, Kajzer-Bonk J, et al. Railway embankments as new habitat for pollinators in an agricultural landscape. PLoS ONE. 2014;9:e101297.

32. Bolger DT, Scott TA, Rotenberry JT. Use of corridor-like landscape structures by bird and small mammal species. Biol Conserv. 2001;102:213-24.
33. Benítez-López A, Alkemade R, Verweij PA. The impacts of roads and other infrastructure on mammal and bird populations: a meta-analysis. Biol Conserv. 2010;143:1307-16.

34. Wagner DL, Ascher JS, Bricker NK. A transmission right-of-way as habitat for wild bees (Hymenoptera: Apoidea: Anthophila) in Connecticut. Ann Entomol Soc Am. 2014;107:1110-20.

35. Tryjanowski P, Sparks TH, Jerzak L, Rosin ZM, Skórka P. A paradox for conservation: electricity pylons may benefit avian diversity in intensive farmland. Conserv Lett. 2014;7:34-40.

36. Clarke DJ, Pearce KA, White JG. Powerline corridors: degraded ecosystems or wildlife havens? Wildl Res. 2006:33:615-26.

37. Trombulak SC, Frissell CA. Review of ecological effects of roads on terrestrial and aquatic communities. Conserv Biol. 2000;14:18-30.

38. Fahrig $L$, Rytwinski T. Effects of roads on animal abundance: an empirical review and synthesis. Ecol Soc. 2009;14:21.

39. Pullin AS, Stewart GB. Guidelines for systematic review in conservation and environmental management. Conserv Biol. 2006;20:1647-56.

40. Conseil général de la Sarthe. La gestion des dépendances vertes routières. 2010. http://www.cg72.fr/iso_upload/La_gestion_ des_D\%C3\%A9pendances vertes_light_0.pdf. Accessed 19 June 2015.

41. Jeusset A, Vargac M, Bertheau Y, Coulon A, Deniaud N, Flamerie De Lachapelle $F$, et al. Can linear transportation infrastructure verges constitute a habitat and/or a corridor for biodiversity in temperate landscapes? A systematic review protocol. Environ Evid. 2016;5. https:// doi.org/10.1186/s13750-016-0056-9.

42. Rytwinski T, Soanes K, Jaeger JAG, Fahrig L, Findlay CS, Houlahan J, et al. How effective is road mitigation at reducing road-kill? A meta-analysis. PLoS ONE. 2016;1 1:e0166941.

43. Coffin AW. From roadkill to road ecology: a review of the ecological effects of roads. J Transp Geogr. 2007;15:396-406.

44. Damarad T, Bekker GJ. COST 341 — habitat fragmentation due to transportation infrastructure: findings of the COST action 341. Luxembour: Office for official publications of the European Communities; 2003. http://www.iene.info/wp-content/uploads/COST341_final_report. pdf. Accessed 15 Mar 2017.

45. R Core Team. R: a language and environment for statistical computing. Vienna: R Found Stat Comput; 2016. https://www.R-project.org/.

46. Lajeunesse MJ, Koricheva J, Gurevitch J, Mengersen K. Recovering missing or partial data from studies: a survey of conversions and imputations for meta-analysis. In: Koricheva J, Gurevitch J, Mengersen K, editors. Handbook of meta-analysis in ecology and evolution. Princeton: Princeton University Press; 2013. p. 195-206.

47. Muskett CJ, Jones MP. The dispersal of lead, cadmium and nickel from motor vehicles and effects on roadside invertebrate macrofauna. Environ Pollut Ser Ecol Biol. 1980;23:231-42.

48. Knapp M, Saska P, Knappová J, Vonička P, Moravec P, Kůrka A, et al. The habitat-specific effects of highway proximity on ground-dwelling arthropods: implications for biodiversity conservation. Biol Conserv. 2013;164:22-9.

49. Samways MJ, Osborn R, Carliel F. Effect of a highway on ant (Hymenoptera: Formicidae) species composition and abundance, with a recommendation for roadside verge width. Biodivers Conserv. 1997;6:903-13.

50. Mallard F. Développement d'une méthode d'évaluation quantitative des effets des projets d'infrastructures de transport terrestre sur les milieux naturels. Chapitre 4-3. Ecole Centrale de Nantes (ECN); 2014. https://tel.archives-ouvertes.fr/tel-01006355/. Accessed 1 Dec 2017.

51. Mallard F. Développement d'une méthode d'évaluation quantitative des effets des projets d'infrastructures de transport terrestre sur les milieux naturels. Chapitre 4-4. Ecole Centrale de Nantes (ECN); 2014 https://tel.archives-ouvertes.fr/tel-01006355/. Accessed 1 Dec 2017.

52. Brückmann SV, Krauss J, Steffan-Dewenter I. Butterfly and plant specialists suffer from reduced connectivity in fragmented landscapes. J Appl Ecol. 2010;47:799-809.

53. Hambäck PA, Summerville KS, Steffan-Dewenter I, Krauss J, Englund G, Crist TO. Habitat specialization, body size, and family identity explain lepidopteran density - area relationships in a cross-continental comparison. Proc Natl Acad Sci. 2007;104:8368-73.

54. Krämer B, Poniatowski D, Fartmann T. Effects of landscape and habitat quality on butterfly communities in pre-alpine calcareous grasslands. Biol Conserv. 2012;152:253-61. 
55. Öckinger E, Bergman K-O, Franzén M, Kadlec T, Krauss J, Kuussaari M, et al. The landscape matrix modifies the effect of habitat fragmentation in grassland butterflies. Landsc Ecol. 2012;27:121-31.

56. Sekar S. A meta-analysis of the traits affecting dispersal ability in butterflies: can wingspan be used as a proxy? J Anim Ecol. 2012;81:174-84.

57. van Swaay C, Warren M, Loïs G. Biotope use and trends of European butterflies. J Insect Conserv. 2006;10:189-209.

58. Bees, wasps \& ants recording society. 2013. http://www.bwars.com/ index.php?q=bee/apidae. Accessed 19 June 2017.

59. Hopfenmüller S, Steffan-Dewenter I, Holzschuh A. Trait-specific responses of wild bee communities to landscape composition, configuration and local factors. PLOS ONE. 2014;9:e104439.

60. Retana J, Arnan X, Cerdá X. A multidimensional functional trait analysis of resource exploitation in European ants. Ecology. 2015;96:2781-93.

61. Bertelsmeier C. Ant profiler-a database of ecological characteristics of ants (Hymenoptera: Formicidae). Myrmecol News. 2013;18:73-6.

62. Martinson HM, Raupp MJ. A meta-analysis of the effects of urbanization on ground beetle communities. Ecosphere. 2013;4:1-24.

63. Homburg K, Homburg N, Schäfer F, Schuldt A, Assmann T. Carabids. org - a dynamic online database of ground beetle species traits (Coleoptera, Carabidae). Insect Conserv Divers. 2014;7:195-205.

64. Lange $M$, Türke $M$, Pašalić E, Boch $S$, Hessenmöller D, Müller J, et al. Effects of forest management on ground-dwelling beetles (Coleoptera; Carabidae, Staphylinidae) in Central Europe are mainly mediated by changes in forest structure. For Ecol Manag. 2014;329:166-76.

65. Pedley SM, Dolman PM. Multi-taxa trait and functional responses to physical disturbance. J Anim Ecol. 2014;83:1542-52.

66. Noordijk J, Schaffers AP, Sỳkora KV. Diversity of ground beetles and spiders in roadside verges with grey hair-grass vegetation. Eur J Entomol. 2008;105:257-65.

67. Le Viol I, Moca J, Julliard R, Kerbiriou C. The contribution of motorway stormwater retention ponds to the biodiversity of aquatic macroinvertebrates. Biol Conserv. 2009;142:3163-71.

68. Gurevitch J, Curtis PS, Jones MH. Meta-analysis in ecology. Adv Ecol Res. 2001;32:199-247.

69. Gleser JJ, Olkin I. Stochastically dependent effect sizes. In: Cooper H, Hedges LV, Valentine JC, editors. The handbook of research synthesis and meta-analysis. 2nd ed. New York: Russell Sage Foundation; 2009.

70. Rosenberg MS. The file-drawer problem revisited: a general weighted method for calculating fail-safe numbers in meta-analysis. Evolution. 2005;59:464-8.

71. Viechtbauer W. Conducting meta-analyses in $\mathrm{R}$ with the metafor package. J Stat Softw. 2010;36:1-48.

72. Fell PE, Warren RS, Curtis AE, Steiner EM. Short-term effects on macroinvertebrates and fishes of herbiciding and mowing Phragmites australisdominated tidal marsh. Northeast Nat. 2006:13:191-212.

73. Gollan JR, de Bruyn LL, Reid N, Smith D, Wilkie L. Can ants be used as ecological indicators of restoration progress in dynamic environments? A case study in a revegetated riparian zone. Ecol Indic. 2011;11:1517-25.

74. Hopwood JL. The contribution of roadside grassland restorations to native bee conservation. Biol Conserv. 2008;141:2632-40.

75. Samways MJ, Sharratt NJ. Recovery of endemic dragonflies after removal of invasive alien trees. Conserv Biol. 2010;24:267-77.

76. Snodgrass GL, Stadelbacher EA. Effect of different grass and legume combinations on spider (Araneae) and ground beetle (Coleoptera: Carabidae) populations in roadside habitats in the Mississippi Delta. Environ Entomol. 1989;18:575-81.

77. Wootton JT. River food web response to large-scale riparian zone manipulations. PLoS ONE. 2012;7:e51839.

78. Cavaillé $P$, Dommanget F, Daumergue N, Loucougaray G, Spiegelberger T, Tabacchi E, et al. Biodiversity assessment following a naturality gradient of riverbank protection structures in French prealps rivers. Ecol Eng. 2013;53:23-30.

79. Dymitryszyn I. The effect of the construction and renovation of a highway bypass in Central Poland on the carabid beetle fauna (Coleoptera: Carabidae). Eur J Entomol. 2014;111:655-62.

80. Paetzold A, Yoshimura C, Tockner K. Riparian arthropod responses to flow regulation and river channelization: riparian arthropod responses to river regulation. J Appl Ecol. 2008;45:894-903.
81. Penone C, Kerbiriou C, Julien J-F, Julliard R, Machon N, Le Viol I. Urbanisation effect on Orthoptera: which scale matters? Insect Conserv Divers. 2012;6:319-27.

82. Scher $\mathrm{O}$, Thièry A. Odonata, Amphibia and environmental characteristics in motorway stormwater retention ponds (Southern France). Hydrobiologia. 2005;551:237-51.

83. Noordijk J, Delille K, Schaffers AP, Sýkora KV. Optimizing grassland management for flower-visiting insects in roadside verges. Biol Conserv. 2009;142:2097-103.

84. Noordijk J, Schaffers AP, Heijerman T, Boer P, Gleichman M, Sýkora KV. Effects of vegetation management by mowing on ground-dwelling arthropods. Ecol Eng. 2010;36:740-50.

85. Russell SA, Thorvilson HG, Phillips SA. Red imported fire ant populations in Texas highway roadsides and rest areas. Southwest Entomol. 2001;26:63-71.

86. Skórka P, Lenda M, Moroń D, Martyka R, Tryjanowski P, Sutherland WJ. Biodiversity collision blackspots in Poland: separation causality from stochasticity in roadkills of butterflies. Biol Conserv. 2015;187:154-63.

87. Ward L, Mill PJ. Habitat factors influencing the presence of adult Calopteryx splendens (Odonata: Zygoptera). Eur J Entomol. 2005;102:47-51.

88. Wynhoff I, van Gestel R, van Swaay C, van Langevelde F. Not only the butterflies: managing ants on road verges to benefit Phengaris (Maculinea) butterflies. J Insect Conserv. 2011;15:189-206.

89. Åström M, Pettersson LB, Öckinger E, Hedin J. Habitat preferences and conservation of the marbled jewel beetle Poecilonota variolosa (Buprestidae). J Insect Conserv. 2013;17:1145-54.

90. Major RE, Christie FJ, Gowing G, Cassis G, Reid CA. The effect of habitat configuration on arboreal insects in fragmented woodlands of southeastern Australia. Biol Conserv. 2003;113:35-48.

91. Major RE, Smith D, Cassis G, Gray M, Colgan DJ. Are roadside strips important reservoirs of invertebrate diversity? A comparison of the ant and beetle faunas of roadside strips and large remnant woodlands. Aust J Zool. 1999:47:611.

92. Noordijk J, Schaffers AP, Heijerman T, Sýkora KV. Using movement and habitat corridors to improve the connectivity for heathland carabid beetles. J Nat Conserv. 2011;19:276-84.

93. Rotholz E, Mandelik Y. Roadside habitats: effects on diversity and composition of plant, arthropod, and small mammal communities. Biodivers Conserv. 2013;22:1017-31.

94. Silverman B, Horn DJ, Purrington FF, Gandhi KJK. Oil pipeline corridor through an intact forest alters ground beetle (Coleoptera: Carabidae) assemblages in southeastern Ohio. Environ Entomol. 2008;37:725-33.

95. Bailey S. Quelle est la contribution des lisières forestières à la structuration des assemblages d'abeilles sauvages dans les paysages agricoles? Chapitre 6. Université d'Orléans; 2014. https://tel.archives-ouvertes.fr/ tel-01203346/. Accessed 11 Sep 2016.

96. Collins B, Foré S. Potential role of pollinators in microhabitat structure within a large population of Echinacea laevigata (Asteraceae). J Torrey Bot Soc. 2009;136:445-56.

97. Hanley ME, Wilkins JP. On the verge? Preferential use of road-facing hedgerow margins by bumblebees in agro-ecosystems. J Insect Conserv. 2015;19:67-74.

98. Osgathorpe LM, Park K, Goulson D. The use of off-farm habitats by foraging bumblebees in agricultural landscapes: implications for conservation management. Apidologie. 2012;43:113-27.

99. Russell KN, Ikerd H, Droege S. The potential conservation value of unmowed powerline strips for native bees. Biol Conserv. 2005; 124:133-48

100. ASF. Evolution de la biodiversité des dépendances autoroutières. Etude comparative 1995-2009. CERA Environnement; 2012

101. Alten B, Bellini R, Caglar SS, Simsek FM, Kaynas S. Species composition and seasonal dynamics of mosquitoes in the Belek region of Turkey. $J$ Vector Ecol. 2000;25:146-54.

102. Braun S, Flückiger W. Increased population of the aphid Aphis pomi at a motorway: part 1—field evaluation. Environ Pollut Ser Ecol Biol. 1984;33:107-20.

103. Kline DL. Seasonal and spatial abundance of Culicoides spp. larvae in roadside salt marsh areas at Yankeetown, Florida. Fla Entomol. 1989;72:111-7.

104. Loch AD, Zalucki MP. Spatial distribution of pink wax scale, Ceroplastes rubens Maskell (Hemiptera: Coccidae), on umbrella trees in 
south-eastern Queensland: the pattern of outbreaks. Aust J Zool. 1996;44:599-609.

105. Russell SA, Thorvilson HG, Phillips SA. Red imported fire ant (Hymenoptera: Formicidae) populations in Texas highway rights-of-way and adjacent pastures. Environ Entomol. 2001;30:267-73.

106. Vogt JT. Nature and severity of imported fire ant (Hymenoptera: Formicidae) infestations on the Mississippi river Levee. Midsouth Entomol. 2009;2:78-83.

107. Mueller EK, Baum KA. Monarch-parasite interactions in managed and roadside prairies. J Insect Conserv. 2014;18:847-53.

108. Ditsworth TM, Butt SM, Beley JR, Johnson CD, Balda RP. Arthropods, plants, and transmission lines in Arizona: community dynamics during secondary succession in a pinyon-juniper woodland. Southwest Nat. 1982;27:167-81.

109. Freitag A, Dischinger C, Cherix D. Formica pratensis (Hyménoptères: Formicidae) dans le canton de Vaud: état des peuplements et importance des talus de routes comme milieu de substitution. Bull Société Vaudoise Sci Nat. 2008;91:47-68.

110. Itzhak MJ-J. Seed harvester and scavenger ants along roadsides in Northern Israel. Zool Middle East. 2008;44:75-82.

111. Larson KA, Harman DM. Subcortical cavity dimension and inquilines of the larval locust borer (Coleoptera: Cerambycidae). Proc Entomol Soc Wash. 2003;105:108-19.

112. Martinez J-JI, Wool D. Sampling bias in roadsides: the case of galling aphids on Pistacia trees. Biodivers Conserv. 2006;15:2109-21.

113. Wilkaniec B, Borowiak-Sobkowiak B, Wilkaniec A, Kubasik W, Kozłowska M, Dolańska-Niedbała E. Aphid migrant activity in refuge habitats of the Wielkopolska agricultural landscape. J Plant Prot Res. 2015;55:69-79.

114. Fleishman E, Austin GT, Brussard PF, Murphy DD. A comparison of butterfly communities in native and agricultural riparian habitats in the Great Basin, USA. Biol Conserv. 1999;89:209-18.

115. Houston WA, Wormington KR, Black RL. Termite (Isoptera) diversity of riparian forests, adjacent woodlands and cleared pastures in tropical eastern Australia. Austral Entomol. 2015;54:221-30.

116. Schipper AM, Wijnhoven S, Leuven RSEW, Ragas AMJ, Jan Hendriks A. Spatial distribution and internal metal concentrations of terrestrial arthropods in a moderately contaminated lowland floodplain along the Rhine River. Environ Pollut. 2008;151:17-26.

117. Brunzel S, Elligsen H, Frankl R. Distribution of the Cinnabar moth Tyria jacobaeae L. at landscape scale: use of linear landscape structures in egg laying on larval hostplant exposures. Landsc Ecol. 2004;19:21-7.

118. Vermeulen HJ. Corridor function of a road verge for dispersal of stenotopic heathland ground beetles Carabidae. Biol Conserv. 1994:69:339-49.

119. Munguira ML, Thomas JA. Use of road verges by butterfly and burnet populations, and the effect of roads on adult dispersal and mortality. J Appl Ecol. 1992;29:316-29.
120. Dallimer M, Rouquette JR, Skinner AMJ, Armsworth PR, Maltby LM, Warren $\mathrm{PH}$, et al. Contrasting patterns in species richness of birds, butterflies and plants along riparian corridors in an urban landscape. Divers Distrib. 2012;18:742-53.

121. Magierowski RH, Davies PE, Read SM, Horrigan N. Impacts of land use on the structure of river macroinvertebrate communities across Tasmania, Australia: spatial scales and thresholds. Mar Freshw Res. 2012;63:762-79.

122. Tagwireyi P, Sullivan SMP. Riverine landscape patch heterogeneity drives riparian ant assemblages in the Scioto River Basin, USA. PLoS ONE. 2015:10:e0124807.

123. Verboven HAF, Uyttenbroeck R, Brys R, Hermy M. Different responses of bees and hoverflies to land use in an urban-rural gradient show the importance of the nature of the rural land use. Landsc Urban Plan. 2014;126:31-41.

124. Eycott AE, Stewart GB, Buyung-Ali LM, Bowler DE, Watts K, Pullin AS. A meta-analysis on the impact of different matrix structures on species movement rates. Landsc Ecol. 2012;27:1263-78.

125. Severns PM. Road crossing behavior of an endangered grassland butterfly, Icaricia icarioides fenderi Macy (Lycaenidae), between a subdivided population. J Lepidopterists Soc. 2008;62:55-8.

126. Vandevelde J-C, Penone C, Julliard R. High-speed railways are not barriers to Pyronia tithonus butterfly movements. J Insect Conserv. 2012;16:801-3.

127. Noordijk J, Prins D, de Jonge M, Vermeulen R. Impact of a road on the movements of two ground beetle species (Coleoptera: Carabidae). Entomol Fenn. 2006;17:276.

128. Hanski I. Habitat loss, the dynamics of biodiversity, and a perspective on conservation. AMBIO J Hum Environ. 2011:40:248-55.

129. Rytwinski T, Fahrig L. Why are some animal populations unaffected or positively affected by roads? Oecologia. 2013;173:1143-56

130. Fahrig L. Rethinking patch size and isolation effects: the habitat amount hypothesis. J Biogeogr. 2013;40:1649-63.

131. Kremen C. Reframing the land-sparing/land-sharing debate for biodiversity conservation. Ann NY Acad Sci. 2015;1355:52-76.

\section{Submit your next manuscript to BioMed Central and we will help you at every step:}

- We accept pre-submission inquiries

- Our selector tool helps you to find the most relevant journal

- We provide round the clock customer support

- Convenient online submission

- Thorough peer review

- Inclusion in PubMed and all major indexing services

- Maximum visibility for your research

Submit your manuscript at www.biomedcentral.com/submit
BioMed Central 